\title{
GENERALIZED COHOMOLOGY OPERATIONS AND $H$-SPACES OF LOW RANK
}

\author{
BY \\ J. R. HUBBUCK
}

Introduction. This note is essentially concerned with the classification of truncated polynomial algebras of height $\geqq 3$ which arise in the $Z_{2}$-cohomology rings of $\mathrm{CW}$-complexes with finite skeletons, where there are not more than five generators and these occur in even dimensions. The precise definition of truncated polynomial algebra is given in $\$ 5$. The approach is to generalize the $K$-theoretic methods of [7]. To assist in this classification, we define a simple generalization of the Steenrod squares for the $Q_{2}$-cohomology rings of finite complexes whose integral homology is free of 2-torsion, where $Q_{2}$ is the ring of rationals with odd denominators. These are not cohomology operations as they only satisfy generalized naturality properties, but under favourable conditions they provide information which it does not seem possible to obtain using either primary or secondary cohomology operations. The treatment of these generalized cohomology operations given in this note is not complete; only those properties are considered which are needed for the immediate applications. In later papers the author hopes to extend both the theory and the applications of these generalized operations as indicated in [13].

All the additive and several of the multiplicative results given in this paper have quite precise analogues for odd primes. These are not considered here, as the most elegant treatment of the corresponding generalized reduced power operations for odd primes involves the use of theorems of J. F. Adams which have not yet been published.

I would like to thank Professor J. F. Adams and Dr. I. M. James for the considerable assistance they have given in the preparation of this paper, which essentially formed a part of the author's doctoral thesis.

1. Statement of results. Let $X$ be a connected finite $\mathrm{CW}$-complex which supports the structure of an $H$-space, that is, for which there exists a continuous map $m: X \times X \rightarrow X$, with unit. A classical theorem of Hopf says that the rational cohomology ring $H^{*}(X, Q)$ is an exterior algebra on odd-dimensional generators. The number of such generators is called the rank of $X$, the dimensions in which they occur, the type of $X$. Thus $U(3)$ has rank 3 and type $(1,3,5)$.

The $H$-space map induces a comultiplication on the ring $H^{*}(X, Q)$ which gives it the structure of an associative, commutative, connected, graded Hopf algebra.

Received by the editors October 31, 1968. 
$H^{*}(X, Q)$ is said to be primitive (with respect to $m$ ), if we can find generators $x_{i}$ for the exterior algebra such that

$$
m^{*}\left(x_{i}\right)=x_{i} \otimes 1+1 \otimes x_{i} \in H^{*}(X \times X, Q) .
$$

Let $X$ be a connected finite complex whose integral homology is free of 2-torsion and which supports the structure of an $H$-space with $H^{*}(X, Q)$ primitive.

THEOREM 1.1. Let rank $X \leqq 5$; then $X$ has the type of an $H$-space which is a product of spaces $S^{1}, S^{7}, \mathrm{SU}(n)$, or $\mathrm{Sp}(n)$ of the same rank.

When rank $X=1$, this is well known; it was first proved in [1]. The result when rank $X=2$ answers questions raised in [2] and [15] $\left({ }^{1}\right)$.

Theorem 1.1 is closely connected with the next theorem. Here and in Theorems 1.3 and $1.4, Y$ is a complex with finite skeletons.

THEOREM 1.2. Let $H^{*}\left(Y, Z_{2}\right)$ be a truncated polynomial algebra of height $\geqq 3$ on not more than five generators all with even dimensions. Then the set of dimensions of the generators is a union of sets taken from $(2),(4),(8),(4,6),(4,8),(4,6,8)$, $(4,8,12),(4,6,8,10),(4,8,12,16),(4,6,8,10,12)$ or $(4,8,12,16,20)$.

For each rational number $q$ let $\nu_{2}(q)$ be the exponent of 2 when $q$ is expressed as a product of powers of distinct primes. A significant step in the proof of Theorem 1.2 is the next rather technical result.

THEOREM 1.3. Let $H^{*}\left(Y, Z_{2}\right)$ be a truncated polynomial algebra of height $\geqq 3$ on generators of dimensions $2 n_{1} \leqq 2 n_{2} \leqq \cdots \leqq 2 n_{m}$. For each $r, 1 \leqq r \leqq m$, let $n_{s}$ and $n_{t}$ be chosen as large as possible with $n_{s}<2 n_{r}$ and $n_{t}<n_{r}\left(n_{t}=0\right.$ if $\left.n_{r}=n_{1}\right)$. Then $\alpha \geqq$ Min $\left(2 n_{r}-n_{s}, n_{r}-n_{t}\right)$, where $\alpha=1$, if $n_{r}$ is odd, and $\alpha=2+\nu_{2}\left(n_{r}\right)$, if $n_{r}$ is even.

This result will follow easily from Theorem 1.4, where $\mathrm{Sq}^{2 q}$ is the Steenrod square.

THEOREM 1.4. Let $Y$ be a $\mathrm{CW}$-complex with finite skeletons and with $H_{*}(Y, Z)$ free of 2-torsion. Suppose that $H^{n+2(q-s)}\left(Y, Z_{2}\right)$ is zero for $1 \leqq s \leqq 2+\nu_{2}(q)$, if $q$ is even, or $H^{n+2(q-1)}\left(Y, Z_{2}\right)$ is zero, if $q$ is odd; then

$$
\mathrm{Sq}^{2 q}: H^{n}\left(Y, Z_{2}\right) \rightarrow H^{n+2 q}\left(Y, Z_{2}\right) \text { is zero. }
$$

The plan of what follows is this. In $\S 2$, an axiomatic description of those properties of the generalized Steenrod squares which are needed, is given, and in $\$ 3$, some applications are made. In $\$ 4$ multiplications are introduced and the long $\S 5$ is concerned with the classification of truncated polynomial algebras. In $\$ 6$, Theorem 1.1 is deduced from the results of $\S 5$.

(1) Dr. R. Douglas and Dr. F. Sigrist have recently announced their independent proof of this result. 
2. The additive structure. Let $Q$ be the field of rational numbers and $Q_{2}$ the subring of rationals with odd denominators. We consider finitely generated $Q_{2}$ modules which possess linear maps $\psi^{k}: M \rightarrow M$, for each integer $k$, and are filtered by submodules

$$
M=M_{0} \supset M_{1} \supset M_{2} \supset \ldots \supset M_{g-1} \supset M_{g}=0,
$$

such that $\psi^{k}\left(M_{i}\right) \subset M_{i}$ for each $i$.

Let $N=\sum_{0 \leqq i \leqq g-1} N_{i}$ be the associated graded module, that is, $N_{i} \cong M_{i} / M_{i+1}$.

$M$ and $\psi^{k}$ will always satisfy the three axioms:

A1. The Splitting Condition. $N$ is a free graded $Q_{2}$-module.

A2. The Commuting Condition. $\psi^{k} \psi^{l}=\psi^{l} \psi^{k}$.

A3. The Integrality Condition. Let $u_{0} \in M_{n}$; then there exists a finite set of elements $u_{i} \in M_{n+i}, 1 \leqq i \leqq h$, such that $u=\sum_{0 \leqq i \leqq h} 2^{-i} u_{i}$ satisfies $\psi^{k}(u)=k^{n} u$ in $M \otimes Q$, for each $k$.

We call such an $M$, a $\psi^{k}$-module over $Q_{2}$. The application is to take $M=K\left(X, Q_{2}\right)$, where $X$ is a finite complex whose integral homology is free of 2-torsion. $K\left(X, Q_{2}\right)$ is unitary $K$-theory with $Q_{2}$ coefficients, defined by taking the tensor product of the integral $K$-theory of [11] with $Q_{2}$. We filter $K\left(X, Q_{2}\right)$ with the CW-filtration. The standard spectral sequence of [11], modified to take into account the coefficients, collapses, since $H^{*}\left(X, Q_{2}\right)$ is torsion free. Thus

$$
H^{2 n}\left(X, Q_{2}\right) \cong K_{2 n}\left(X, Q_{2}\right) / K_{2 n+1}\left(X, Q_{2}\right)
$$

Set $M_{i}=K_{2 i}\left(X, Q_{2}\right)=K_{2 i-1}\left(X, Q_{2}\right)$ and $\psi^{k}$ as the Adams operators of [4]. Then $N_{i} \cong M_{i} / M_{i+1} \cong H^{2 i}\left(X, Q_{2}\right)$.

LEMMA 2.1. $M=K\left(X, Q_{2}\right)$ is a $\psi^{k}$-module over $Q_{2}$.

Proof. The only point which needs to be discussed is A3. The other facts are either trivial or well known. Given $u_{0} \in M_{n}$, we show that there exist elements $u_{i} \in M_{n+i}, 1 \leqq i \leqq h$, such that $u=\sum_{0 \leqq i \leqq h} 2^{-i} u_{i}$ satisfies the condition ch $u$ $\in H^{2 n}(X, Q)$. Here ch is the extension of the Chern character to $K(X, Q)$. The result will then follow from $\S 2$ of [7] for if $K(X, Q)$ and $H^{\text {even }}(X, Q)$ are identified by means of the Chern character, $H^{2 n}(X, Q)$ is an eigenspace of $\psi^{k}$ corresponding to the eigenvalue $k^{n}$.

Therefore suppose that there exists an element $u$ of the required form such that ch $u \in H^{2 n}(X, Q) \bmod \sum_{i \geqq m} H^{2 i}(X, Q)$. This is certainly true for $m=n+1$ and so as inductive hypothesis suppose that it is true for $m<q=n+s$. Theorem 1 of [3] implies that $2^{s} \mathrm{ch}_{2 q} u \in i_{*} H^{2 q}\left(X, Q_{2}\right)$, where $i: Q_{2} \rightarrow Q$ is the inclusion map and $\mathrm{ch}_{2 q}$ is the $2 q$ th component of $\mathrm{ch}$. Thus there exists an element $w_{q} \in K_{2 q}\left(X, Q_{2}\right)$ with $\operatorname{ch}_{2 q} w_{q}=2^{s} \operatorname{ch}_{2 q} u$. Replace $u_{s}$ by $u_{s}-w_{q}$; then with the new $u_{s}$, ch $u \in H^{2 n}(X, Q)$ $\bmod \sum_{i \geqq q} H^{21}(X, Q)$, which completes the inductive step and therefore the proof of the lemma.

We return to the general situation. 
LEMMA 2.2. Let $M=M^{1} \oplus M^{2}$ be a $\psi^{k}$-module over $Q_{2}$ with $\psi^{k}\left(M^{1}\right) \subset M^{1}$ for each $k$; then the quotient module $M^{2}$ is a $\psi^{k}$-module over $Q_{2}$.

The proof is clear.

The next three lemmas are all simple consequences of the axioms A1, A2 and A3.

LEMMA 2.3. $M_{n} \cap\left(M_{n+s} \otimes Q\right)=M_{n+s}$.

Proof. $M_{n+s}$ is a direct summand in $M_{n}$ and so the result follows.

LEMMA 2.4. Let $u_{0} \in M_{n}$; then $\psi^{k}\left(u_{0}\right)=k^{n} u_{0} \bmod M_{n+1}$.

Proof. $\psi^{k}\left(u_{0}\right)=k^{n} u_{0} \bmod M_{n+1} \otimes Q$, by A3. The result follows from Lemma 2.3 since $M$ is free.

Let $I_{s}: M_{s} \rightarrow N_{s}$ be the quotient map. We now explain why Al is called the splitting condition.

LEMMA 2.5. There exists an isomorphism $J: N \rightarrow M$ of filtered $Q_{2}$-modules such that $J\left(N_{s}\right) \subset M_{s}$ and the composition of $J: N_{s} \rightarrow M_{s}$ with $I_{s}: M_{s} \rightarrow N_{s}$ is the identity map on $N_{s}$.

Proof. Let $x_{1}, x_{2}, \ldots, x_{t}$ be a homogeneous base for $N$ over $Q_{2}$. Let $u_{1}, u_{2}, \ldots, u_{t}$ be any elements of $M$ such that $I_{s}\left(u_{i}\right)=x_{i}$, where $x_{i} \in N_{s}, 1 \leqq i \leqq t$. Then the linear map defined by setting $J\left(x_{i}\right)=u_{i}, 1 \leqq i \leqq t$ is an isomorphism of the required type.

Let $J: N \rightarrow M$ be a particular choice for the splitting given by Lemma 2.5 . The linear maps $\Phi_{J}^{k}: N \rightarrow N$ are defined by requiring that the diagram (2.1) commutes.

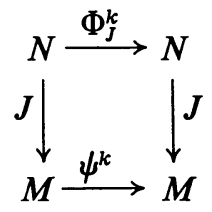

LEMMA 2.6. Let $x_{0} \in N_{n}$; then $\Phi_{J}^{k}\left(x_{0}\right)=k^{n} x_{0} \bmod \sum_{i>n} N_{i}$.

Proof. This follows from Lemma 2.4 .

LEMMA 2.7. $\Phi_{J}^{k} \Phi_{J}^{l}=\Phi_{J}^{l} \Phi_{J}^{k}$.

Proof. This is an immediate consequence of the definitions and A2.

THEOREM 2.8. (i) Let $x_{0} \in N_{n}$; then there exist elements $x_{i}=x_{i}\left(J, x_{0}\right) \in N_{n+i}$, $1 \leqq i \leqq t$, such that $x=\sum_{0 \leqq i \leqq t} 2^{-i} x_{i}$ satisfies $\Phi_{J}^{k}(x)=k^{n} x$ in $N \otimes Q$.

(ii) $x_{i}=x_{i}\left(J, x_{0}\right)$ is unique for $0 \leqq i \leqq t$.

Proof. (i) $J\left(x_{0}\right) \in M_{n}$ and so by A3 there exist $u_{i} \in M_{n+i}, 1 \leqq i \leqq h$, such that $u=\sum_{0 \leqq i \leqq h} 2^{-i} u_{i}$ satisfies $\psi^{k}(u)=k^{n} u$, where $u_{0}=J\left(x_{0}\right)$. Choose $x_{i}^{\prime} \in \sum_{j \geqq_{n+i}} N_{j}$ with $J\left(x_{i}^{\prime}\right)=u_{i}$, and set $x=\sum_{0 \leqq i \leqq h} 2^{-i} x_{i}^{\prime}$. Clearly we may write $x$ in the form $\sum_{0 \leqq i \leqq t} 2^{-i} x_{i}$, where $x_{i} \in N_{n+i} . J\left\{\Phi_{J}^{k}(x)-k^{n} x\right\}=\psi^{k}(u)-k^{n} u=0$ and so $\Phi_{J}^{k}(x)=k^{n} x$ in $N \otimes Q$ as required. 
(ii) Let $y_{i}$ and $z_{i}$ be two choices for the $x_{i}$, so $y=\sum_{0 \leqq i \leqq s} 2^{-i} y_{i}$ and $z=\sum_{0 \leqq i \leqq r} 2^{-i} z_{i}$ satisfy $\Phi_{J}^{k}(y)=k^{n} y$ and $\Phi_{J}^{k}(z)=k^{n} z$. Let $i=r$ be the smallest $r$ with $y_{r} \neq z_{r}$; then $r>0$, by Lemma 2.6 .

$$
\begin{aligned}
\Phi_{J}^{2}(y-z) & =\Phi_{J}^{2}\left\{2^{-r}\left(y_{r}-z_{r}\right)\right\} \bmod \sum_{i>r} N_{n+i} \otimes Q \\
& =2^{n-r}\left(y_{r}-z_{r}\right) \bmod \sum_{i>r} N_{n+i} \otimes Q .
\end{aligned}
$$

But $\Phi_{J}^{2}\left(y_{r}-z_{r}\right)=2^{n+r}\left(y_{r}-z_{r}\right) \bmod \sum_{i>r} N_{n+i} \otimes Q$, by Lemma 2.6. Therefore $y_{r}=z_{r}$.

Define homomorphisms $S_{f}^{q}: N_{n} \rightarrow N_{n+q}$ by setting $S_{9}^{q} x_{0}=x_{q}$. These are the generalized Steenrod squares of the Introduction, and it will be shown that they satisfy properties somewhat analogous to the usual Steenrod squares.

Let $S_{J}(t)=\sum_{q \geq 0} S_{J}^{q} t^{q}$ and let $Q_{J}(t)=\sum_{q \geqq 0} Q_{J}^{q} t^{q}$ be its formal inverse. Also let $R_{J}(t ; k)=\sum_{q \geqq 0} R_{J}^{q}(k) t^{q}=S_{J}\left(2^{-1} t\right) Q_{J}\left(k 2^{-1} t\right)$. When there can be no confusion concerning which splitting $J$ we are using, we omit the suffix $J$ in all the above notations.

LEMMA 2.9. When $\Phi^{k}$ is restricted to $N_{n}$,

$$
\Phi^{k}=k^{n} R(1, k)=k^{n} \sum_{q \geqq 0} R^{q}(k) .
$$

Proof. Let $x_{0} \in N_{n}$; then $Q^{j}\left(x_{0}\right) \in N_{n+j}$. Therefore

$$
\Phi^{k}\left\{S\left(2^{-1}\right) Q^{j}\left(x_{0}\right)\right\}=k^{n+j} S\left(2^{-1}\right) Q^{j}\left(x_{0}\right) \equiv B_{j} .
$$

Considering $\sum_{j \geqq 0} 2^{-j} B_{j}$, we obtain

$$
\begin{aligned}
\Phi^{k}\left(x_{0}\right) & =k^{n} S\left(2^{-1}\right)\left\{\sum_{i \geqq 0} 2^{-i} k^{i} Q^{i}\left(x_{0}\right)\right\} \\
& =k^{n} S\left(2^{-1}\right) Q\left(2^{-1} k\right)\left(x_{0}\right)=k^{n} R(1 ; k)\left(x_{0}\right) .
\end{aligned}
$$

The $S^{q}$ have been constructed from the $\psi^{k}$, but Lemma 2.9 enables us to reverse this process. Therefore, given homomorphisms $S^{q}: N_{n} \rightarrow N_{n+q}$, from them we can construct $\Phi^{k}$ and hence $\psi^{k}$.

THEOREM 2.10. $M$ is a $\psi^{k}$-module over $Q_{2}$ if and only if $k^{n} R^{q}(k)\left(N_{n}\right) \subset N_{n+q}$, for all $n, q$ and $k$.

Proof. The necessity of the condition follows immediately from the last lemma. Conversely if $k^{n}\left(R^{q}(k)\right)\left(N_{n}\right) \subset N_{n+q}, \Phi^{k}\left(x_{0}\right)=k^{n} R(1 ; k)\left(x_{0}\right)$, for $x_{0} \in N_{n}$, is well defined and is contained in $\sum_{i} \geq_{n} N_{i}$. Therefore $\psi^{k}$ is filtration preserving. Also

$$
\begin{aligned}
\Phi^{m} \Phi^{k}\left(x_{0}\right) & =k^{n}\left\{R(1 ; m)\left(m^{n} R^{0}(k)+m^{n+1} R^{1}(k)+\cdots+m^{n+s} R^{s}(k)+\cdots\right)\right\}\left(x_{0}\right) \\
& =k^{n} m^{n}\{R(1 ; m) R(m ; k)\}\left(x_{0}\right)=(k m)^{n} R(1 ; k m)\left(x_{0}\right) .
\end{aligned}
$$

Therefore $\psi^{m} \psi^{k}\left(J\left(x_{0}\right)\right)=\psi^{m k}\left(J\left(x_{0}\right)\right)$, which is sufficient to prove A2. 
Finally we consider A3:

$$
\Phi^{k}\left(S^{q}\left(x_{0}\right)\right)=k^{n+q} R(1 ; k) S^{q}\left(x_{0}\right)=k^{n} R(1 ; k) k^{q} S^{q}\left(x_{0}\right) .
$$

Therefore,

$$
\Phi^{k}\left(S\left(2^{-1}\right)\left(x_{0}\right)\right)=k^{n} R(1 ; k) S\left(2^{-1} k\right)\left(x_{0}\right)=k^{n} S\left(2^{-1}\right)\left(x_{0}\right) .
$$

This proves A3 for all elements of the form $J\left(x_{0}\right)$ with $x_{0} \in N_{n}$, and the general result quickly follows.

Theorem 2.10 implies that the component of $\Phi^{k}\left(x_{0}\right)$ in dimension $n+q$ is

$$
k^{n} R^{q}(k)\left(x_{0}\right)=k^{n} 2^{-q}\left(S^{q}+k S^{q-1} Q^{1}+\cdots+k^{q} Q^{q}\right)\left(x_{0}\right)
$$

where $x_{0} \in N_{n}$. Therefore we set $T_{J}^{q}(k)=2^{q} R_{J}^{q}(k)$. It follows from the definition of $Q^{q}$ that

$$
T^{q}(k)=\left(1-k^{q}\right) S^{q}+\left(k-k^{q}\right) S^{q-1} Q^{1}+\cdots+\left(k^{q-1}-k^{q}\right) S^{1} Q^{q-1} .
$$

The following corollary to Theorem 2.10 is immediate.

Corollary 2.11 (Generalized Adem Relations). Let $k$ be an odd integer. Then $T_{J}^{q}(k)=0 \bmod 2^{q}$.

This result is the main technical tool of this paper, but before discussing it one further relation is established.

LEMMA 2.12. $T^{q}(k)=\left(1-k^{q}\right) S^{q}-\sum_{1 \leqq i \leqq q-1} k^{i} T^{q-i}(k) S^{i}$.

Proof. $R(t ; k)=S\left(2^{-1} t\right) Q\left(2^{-1} k t\right)$ and so $R(t ; k) S\left(2^{-1} k t\right)=S\left(2^{-1} t\right)$. Equating the coefficients of $t^{q}$, the result follows.

Now consider Corollary 2.11. Let $k$ be an odd integer and first take $q=2$. Then $T^{2}(k)=0 \bmod 4$, or $S^{2}+k S^{1} Q^{1}+k^{2} Q^{2}=0 \bmod 4$ and so, by the definition of $Q^{2}$, $\left(1-k^{2}\right) S^{2}+k(1-k) S^{1} Q^{1}=0 \bmod 4$. Taking $k=-1 \bmod 4$ it follows that $S^{1} Q^{1}$ $=0 \bmod 2$ or $S^{1} S^{1}=0 \bmod 2$. Now let $q=3$ and obtain $T^{3}(k)=0 \bmod 8$, or

$$
\left(1-k^{3}\right) S^{3}+k\left(1-k^{2}\right) S^{2} Q^{1}+k^{2}(1-k) S^{1} Q^{2}=0 \bmod 8 .
$$

Again taking $k=-1 \bmod 4$, deduce that $S^{3}+S^{1} Q^{2}=0 \bmod 4$ and hence $S^{3}$ $=S^{1} S^{2} \bmod 2$. If we put $q=4$, we would obtain the relations $S^{1} S^{3}=0 \bmod 2$ and $S^{2} S^{2}=S^{3} S^{1} \bmod 2$. These of course correspond to certain Adem relations in the mod 2 Steenrod algebra, $\mathscr{A}(2)$. The identification of $S^{q} \bmod 2$ with $\mathrm{Sq}^{2 q}$ will follow from the next lemma. However though $\mathscr{A}(2) \cong \mathscr{A}(2) /\left(\mathrm{Sq}^{1}\right)$, Corollary 2.11 does not imply all the Adem relations. It is not true for example that $S^{1} S^{4}+S^{4} S^{1}$ $=S^{2} S^{3} \bmod 2$. To see this let $M=Q_{2} \oplus Q_{2} \oplus Q_{2} \oplus Q_{2} \oplus Q_{2}$ with $N_{i}=Q_{2}$ generated by $x_{i-4}$ for $i=5,7,8,9$ or 10 and $N_{i}=0$ otherwise. Set (a) $S^{1} x_{3}=x_{4}, S^{1} x_{i}=0$ otherwise; (b) $S^{2} x_{1}=x_{3}, S^{2} x_{4}=x_{6}$ but $S^{2} x_{3}=0$; (c) $S^{3} x_{1}=x_{4}$ and $S^{3} x_{3}=0$; (d) $S^{4} x_{1}=0$; and (e) $S^{5} x_{1}=0$. It is a simple matter to check that the conditions of Theorem 2.10 are satisfied, but $S^{1} S^{4}+S^{4} S^{1} \neq S^{2} S^{3} \bmod 2$. We shall not consider 
here precisely which relations are obtained. If one defines $\mathscr{A}^{\prime}(2)$ using the relations mod 2 implied by Corollary 2.11 in a manner analogous to the definition of the mod 2 Steenrod algebra using the Adem relations, then one obtains an associative, graded algebra, generated as an algebra by $S^{2^{i}} \bmod 2, i \geqq 0$; but as we have seen above there are fewer relations connecting these generators than in the Steenrod algebra.

LEMMA 2.13. (a) Let $u_{0} \in M_{n}$; then there exist elements $v_{i} \in M_{n+i}, 0 \leqq i \leqq n$, such that $\psi^{2}\left(u_{0}\right)=\sum_{0 \leqq i \leqq n} 2^{n-i} v_{i}$ and $u_{0}-v_{0} \in M_{n+1}$.

(b) Let $x_{0} \in N_{n}$; then there exist elements $y_{i} \in N_{n+i}, 0 \leqq i \leqq s$ for some $s$, such that $\Phi_{J}^{2}\left(x_{0}\right)=\sum_{0 \leqq i \leqq s} 2^{n-i} y_{i}$, where $x_{0}=y_{0}$ and $2^{n-i} y_{i} \in N_{n+i}$.

(c) $S_{J}^{q}\left(x_{0}\right)=y_{q} \bmod 2,0 \leqq i \leqq s$.

(d) If $x_{0}=I_{n}\left(u_{0}\right)$, then $y_{i}=I_{n+i}\left(v_{i}\right) \bmod 2,0 \leqq i \leqq n$.

Proof. Part (b) follows from Lemma 2.9, part (a) follows from part (b) and part (c) follows from Lemma 2.9. It remains to prove (d).

Since $I_{n}\left(u_{0}\right)=x_{0}, \quad J\left(x_{0}\right)=u_{0}+u_{0}^{\prime}$, where $u_{0}^{\prime} \in M_{n+1}$. Suppose that $\psi^{2}\left(u_{0}^{\prime}\right)$ $=\sum_{0 \leqq i \leqq n+1} 2^{n+1-i} w_{i+1}$, where $w_{i} \in M_{n+i} ;$ then

$$
\psi^{2}\left(J\left(x_{0}\right)\right)=\sum_{0 \leqq i \leqq n} 2^{n-i}\left(v_{i}+2 w_{i+1}\right)+w_{n+2} .
$$

Therefore $2^{n-i} J\left(y_{i}\right)=2^{n-i} v_{i} \bmod \left\{2^{n+1-i} M+M_{n+1-i}\right\}$, from which it follows that $y_{i}=I_{n+i}\left(v_{i}\right) \bmod 2$.

It is a consequence of (c) and (d) that $S_{J}^{q}\left(x_{0}\right) \bmod 2$ is independent of the particular choice of $J$ for $0 \leqq i \leqq n$. Also from (b) and (c) it follows that $S_{j}^{q}\left(x_{0}\right)=$ $0 \bmod 2$, for $q>n$. If we consider the particular case of a $\psi^{k}$-module over $Q_{2}$ described in Lemma 2.1, it follows from the results of [9], see especially Proposition 5.6, that $S^{q} \bmod 2$, restricted to $N \otimes Z_{2}$, is just the Steenrod square $\mathrm{Sq}^{2 q}$. This identification can, of course, also be obtained from [3].

3. Applications. In this section some theorems are proved using the results of the last section. In particular, the proof of Theorem 1.4 is given.

LEMMA 3.1. Let $k$ be an odd integer; then

$$
\begin{aligned}
\nu_{2}\left(k^{t}-1\right) & \geqq 1, & & \text { if } t \neq 0 \bmod 2 \\
& \geqq 2+\nu_{2}(t), & & \text { if } t=0 \bmod 2 .
\end{aligned}
$$

Moreover the equality is attained in the following cases,

(i) $t$ is even and $k$ is a generator of $G_{8} /( \pm 1)$,

(ii) $t$ is odd and $k$ is a generator of $G_{4}$, where $G_{n}$ is the group of units in the integers modulo $n$.

Proof. For a detailed proof and explanation of this lemma, we refer to $\$ 2$ of [5].

Let $\mathrm{Sr}^{i}$ be written for $S^{i}$ mod 2 . We remind the reader of the following result. 
Lemma 3.2. Let $N_{i}=0$, unless $i \leqq n$ or $i \geqq 2 n$. Then $\operatorname{Sr}^{n}: N_{n} \otimes Z_{2} \rightarrow N_{2 n} \otimes Z_{2}$ is zero, unless $n=1,2$ or 4 .

Proof. Corollary 2.11 and Lemma 2.12 imply that $\left(1-k^{n}\right) S^{n}\left(N_{n}\right)=0 \bmod 2^{n}$ for odd $k$. The result follows from Lemma 3.1, since $2+v_{2}(n) \geqq n$ implies that $n=1,2$ or 4.

We now generalize this result to obtain Theorem 1.4.

THEOREM 3.3. Suppose that if $q$ is even $N_{n+q-s}$ is zero for $1 \leqq s \leqq 2+\nu_{2}(q)$, and if $q$ is odd $N_{n+q-1}$ is zero. Then $\mathrm{Sr}^{q}: N_{n} \otimes Z_{2} \rightarrow N_{n+q} \otimes Z_{2}$ is zero.

Proof. Write $q-t \leqq s \leqq q-1$ for the two ranges mentioned above where $N_{n+s}$ is zero. Lemma 2.12 implies that on $N_{n}$

$$
T^{q}(k)=\left(1-k^{q}\right) S^{q}-\sum_{1 \leqq i \leqq q-t-1} k^{i} T^{q-i}(k) S^{i},
$$

and if $k$ is odd Corollary 2.11 says that $T^{i}(k)=0 \bmod 2^{i}$. The result now follows from Lemma 3.1 as did Lemma 3.2.

In particular, if we consider the case of a $\psi^{k}$-module discussed in Lemma 2.1, and use the identification of $S^{i} \bmod 2$ and $\mathrm{Sq}^{2 i}$ mentioned after Lemma 2.13, this is just Theorem 1.4, at least for finite complexes.

The particular choice of the splitting $J$ has played no part in the argument so far. We now show how certain simplifications can be effected by restricting the choice of $J$.

Let $x_{1}, x_{2}, \ldots, x_{m}$ be a homogeneous base for $N$, and let $x_{t}$ be an element of this base of dimension $n$. Then we shall choose $J$ such that if $\Phi_{J}^{2}\left(x_{t}\right)=\sum_{1 \leqq i \leqq m} a_{i} x_{i}$, then $2^{n}$ divides a nonzero $a_{i}$ only if $i=t$. Suppose that $\Phi_{J}^{2}\left(x_{t}\right)$ is of the required form $\bmod \sum_{i>n+r} N_{i}$. This is certainly true for $r=0$ by Lemma 2.6, and so as induction hypothesis assume that it is true for $r<q$. Let $a_{s}$ be divisible by $2^{n}$ where $x_{s}$ has dimension $n+q$. The elements $J\left(x_{i}\right), 1 \leqq i \leqq m$, form a base for $M$ over $Q_{2}$, and the coefficient of $J\left(x_{s}\right)$ in $\psi^{2}\left\{J\left(x_{t}\right)+\lambda J\left(x_{s}\right)\right\}$, when expressed in terms of this base, is $a_{s}+2^{n+a} \lambda$, using Lemma 2.4. Therefore if we define a new splitting by setting $K\left(x_{t}\right)=J\left(x_{t}\right)+\lambda J\left(x_{s}\right)$ and $K\left(x_{i}\right)=J\left(x_{i}\right)$ otherwise, and let $\lambda=\left(2^{n}-2^{n+q}\right)^{-1} a_{s}$, which lies in $Q_{2}$, the coefficient of $K\left(x_{s}\right)$ in $\psi^{2}\left(K\left(x_{t}\right)\right)$ is zero, when expressed in terms of the base $K\left(x_{i}\right), 1 \leqq i \leqq m$. We can perform this operation simultaneously for each $x_{s}$ of dimension $n+q$ with corresponding $a_{s}$ divisible by $2^{n}$. Therefore we have produced a splitting with $\Phi_{K}^{2}\left(x_{t}\right)$ of the required form $\bmod \sum_{i>n+q} N_{i}$, which completes the induction step and proves the result.

As an application of this idea, consider the following situation. Let $\tilde{x}_{1}, \tilde{x}_{2}, \ldots, \tilde{x}_{s}$ be a base for the vector subspace of $N \otimes Z_{2}$ which is the kernel of $\operatorname{Sr}^{1}$ and extend this base over the whole of $N \otimes Z_{2}, \tilde{x}_{1}, \ldots, \tilde{x}_{s}, \tilde{y}_{1}, \ldots, \tilde{y}_{t}$. Since $Q_{2}$ is a local ring, we may lift this base to a base of $N, x_{1}, \ldots, x_{s}, y_{1}, \ldots, y_{t}$. The above modification of the splitting is performed simultaneously for each element of the base, which is clearly possible. Now $\Phi_{J}^{2}=2^{n} \sum_{i \geqq 0} R_{J}^{i}(2)$ and $R_{J}^{1}(2)=2^{-1}(1-2) S_{J}^{1}$ when we restrict 
to $N_{n}$. It follows that $S_{J}^{1}\left(x_{i}\right)=0,1 \leqq i \leqq s$, and $S_{J}^{1}\left(y_{j}\right)=\sum_{1 \leqq i \leqq s} \alpha_{i j} x_{i}+\sum_{1 \leqq i \leqq t} \beta_{i j} y_{i}$, where all nonzero $\alpha_{i j}$ and $\beta_{i j}$ are not divisible by 2 . But $S_{J}^{1} S_{J}^{1}=0 \bmod 2$ and so $S_{J}^{1}\left\{\sum_{1 \leqq i \leqq t} \beta_{i j} y_{i}\right\}$ is zero mod 2 which is impossible unless all $\beta_{i j}$ are zero.

In particular, we have shown that there exists a splitting $J$ such that $S_{J}^{1} S_{J}^{1}=0$.

LEMMA 3.4. Let $\nu_{2}(q)=1$, but $q \neq 2$. If $\mathrm{Sr}^{q-3} \tilde{x}=\mathrm{Sr}^{q-2} \tilde{x}=\mathrm{Sr}^{q-1} \tilde{x}=0$ in $N \otimes Z_{2}$, then $\mathrm{Sr}^{a} \tilde{x}=0$.

Proof. Let $x \in N$ be a $Q_{2}$ representative for $\tilde{x}$. Corollary 2.11 and Lemma 2.12 imply that for odd $k$

$$
\begin{aligned}
\left(1-k^{q}\right) S^{q} x & =k^{q-1} T^{1}(k) S^{q-1} x+k^{q-2} T^{2}(k) S^{q-2} x \bmod 16, \\
T^{2}(k) & =\left(1-k^{2}\right) S^{2}-k(1-k) S^{1} S^{1}
\end{aligned}
$$

and, by the remarks preceding this lemma, we may assume that $S^{1} S^{1}=0$. Therefore

$$
\left(1-k^{q}\right) S^{q} x=k^{q-1}(1-k) S^{1} S^{q-1} x \bmod 16
$$

and by alternately putting $k=3$ and $k=5$, it is apparent that this is only possible if $S^{q} x=0 \bmod 2$, that is, $\mathrm{Sr}^{q} \tilde{x}=0$.

The last few results would suggest that we consider secondary and higher order cohomology operations, and in general try and establish a more invariant approach to the $S_{j}^{q}$. Such questions are left for another occasion.

4. The multiplicative structure. Suppose now that $M$ is not just a $\psi^{k}$-module over $Q_{2}$, but in addition it also possesses a multiplication. More precisely let $M$ satisfy the three further axioms:

A4. $M$ is a commutative filtered ring, that is, $M$ is a commutative ring and $M_{i} \cdot M_{j}$ $\subset M_{i+j}$.

A5. $\psi^{k}$ is a ring homomorphism for each integer $k$.

A6. $\psi^{2}(u)=u^{2} \bmod 2$, for each $u \in M$.

We call such an $M$ a multiplicative $\psi^{k}$-module over $Q_{2}$. The application is once more that described in Lemma 2.1. Tensor product of vector bundles induces the structure of a commutative, filtered ring on $K\left(X, Q_{2}\right)$ and $\mathrm{A} 5$ and $\mathrm{A} 6$ are well known properties of the Adams operators.

LEMMA 4.1. $M=K\left(X, Q_{2}\right)$ is a multiplicative $\psi^{k}$-module over $Q_{2}$.

Returning to the general situation, the following result is clear.

LEMMA 4.2. Let $M=M^{1} \oplus M^{2}$ be a multiplicative $\psi^{k}$-module over $Q_{2}$, where $M^{1}$ is an ideal in $M$ such that $\psi^{k}\left(M^{1}\right) \subset M^{1}$ for each $k$. Then the quotient module $M^{2}$ is a multiplicative $\psi^{k}$-module over $Q_{2}$.

In general it will not be possible to find a splitting $J: N \rightarrow M$ which is a ring isomorphism, where $N$ has its induced graded ring structure. The following result however does hold. 
LEMMA 4.3. $N \otimes Q$ and $M \otimes Q$ are isomorphic as rings.

Proof. Let $x_{1}, x_{2}, \ldots, x_{m}$ be a homogeneous base for $N$ over $Q_{2}$. If $J$ is any splitting, set $u_{i}=J\left(x_{i}\right), 1 \leqq i \leqq m$, and then the $u_{i}$ form a base for $M$. Define the linear map $H: N \rightarrow M \otimes Q$ by setting $H\left(x_{i}\right)=\bar{u}_{i}, 1 \leqq i \leqq m$, where $\bar{u}_{i}$ is the element of $M \otimes Q$ associated with $u_{i}$ by A3. It is easy to check that $H$ is well defined and is independent of both $J$ and the choice of base. But $J\left(x_{i}\right) J\left(x_{j}\right)=J\left(x_{i} x_{j}\right) \bmod M_{s+t+1}$, where $x_{i} \in N_{s}$ and $x_{j} \in N_{t}$. Since $\psi^{k}$ is a ring homomorphism, this implies $\bar{u}_{i} \cdot \bar{u}_{j}$ $=\bar{u}_{i} u_{j}$. Thus $H$ is a ring homomorphism and its extension over $N \otimes Q$ is a ring isomorphism.

The most general result involving a splitting is the next lemma.

LEMMA 4.4.

$$
J\left\{S_{J}\left(2^{-1}\right)(x y)\right\}=J\left\{S_{J}\left(2^{-1}\right)(x)\right\} J\left\{S_{J}\left(2^{-1}\right)(y)\right\} \quad \text { in } M \otimes Q
$$

and

$$
Q_{J}\left(2^{-1}\right)\left\{J^{-1}(J(x) \cdot J(y))\right\}=\left\{Q_{J}\left(2^{-1}\right)(x)\right\} \cdot\left\{Q_{J}\left(2^{-1}\right)(y)\right\} \quad \text { in } N \otimes Q .
$$

Proof. Let $J(x)=u$ and $J(y)=v$. Then in the notation of the proof of the last lemma $\bar{u} \cdot \bar{v}=\overline{u v}$. But $J\left\{S_{J}\left(2^{-1}\right)(x)\right\}=\bar{u}$, etc. and the first statement follows.

For the second statement notice that

$$
J\left\{S_{J}\left(2^{-1}\right)\left(Q_{J}\left(2^{-1}\right)(x) \cdot Q_{J}\left(2^{-1}\right)(y)\right)\right\}=J\left\{S_{J}\left(2^{-1}\right) Q_{J}\left(2^{-1}\right)(x)\right\} J\left\{S_{J}\left(2^{-1}\right) Q_{J}\left(2^{-1}\right)(y)\right\},
$$

by the first part, and the right-hand side is just $J(x) \cdot J(y)$. Hence

$$
Q_{J}\left(2^{-1}\right) J^{-1}(J(x) \cdot J(y))=\left\{Q_{J}\left(2^{-1}\right)(x)\right\}\left\{Q_{J}\left(2^{-1}\right)(y)\right\} .
$$

COROllary 4.5 (CARTAN FORMUlaE). (a) If $J$ is a ring isomorphism,

$$
S_{J}^{q}(x \cdot y)=\sum_{i+j=q} S_{J}^{i}(x) \cdot S_{J}^{j}(y) .
$$

(b) If $J(x \cdot y)=J(x) \cdot J(y), Q_{J}^{q}(x \cdot y)=\sum_{i+j=q} Q_{J}^{i}(x) \cdot Q_{J}^{j}(y)$.

Proof. This follows almost immediately from Lemma 4.4.

LEMMA 4.6. Let $\tilde{x} \in N_{n} \otimes Z_{2}$; then $\operatorname{Sr}^{n}(\tilde{x})=\tilde{x}^{2}$.

Proof. Let $x$ represent $\tilde{x}$ over $Q_{2}$. In the notation of Lemma 2.13, $v_{n}=\{J(x)\}^{2}$ $\bmod 2$ by A6, for any splitting $J$ such that $J(x)=u_{0}$. Therefore $y_{n}=I_{2 n}\{J(x)\}^{2} \bmod 2$, and so $y_{n}=x^{2} \bmod 2$. The result follows from Lemma 2.13 (c).

In fact, for most of this paper, it will be sufficient to restrict attention to those $M$ for which there exists a splitting which is a ring isomorphism.

LEMMA 4.7. Let $J: N \rightarrow M$, be a ring isomorphism; then $\Phi^{k}$ is a ring homomorphism.

LEMMA 4.8. Let $J: N \rightarrow M$, be a ring isomorphism; then $\Phi^{2}(x)=x^{2} \bmod 2$ for all $x \in N$. 
The proofs of these last two lemmas follow immediately from the axioms.

5. Truncated polynomial algebras. Let $N$ be a connected graded ring, generated by homogeneous elements $1, x_{1}, x_{2}, \ldots, x_{m}$ of dimensions $0<n_{1} \leqq n_{2} \leqq \cdots \leqq n_{m}$, where $x_{1}^{\nu_{1}} x_{2}^{\nu_{2}} \cdots x_{m}^{\nu_{m}}$ is zero if and only if $\sum_{1 \leqq i \leqq m} \nu_{i} \geqq k$, and there are no other relations among the $x_{i}$. We say that $N$ is a truncated polynomial algebra of height $k$ on generators $x_{1}, x_{2}, \ldots, x_{m}$.

The height of the element $x_{1}^{\mu_{1}} x_{2}^{\mu_{2}} \cdots x_{m}^{\mu_{m}}$ is $\sum_{1 \leqq i \leqq m} \mu_{i}$. Elements which can be expressed as a sum of elements of height greater than or equal to 2 are said to be decomposable.

LEMMA 5.1. There exists a splitting $J: N \rightarrow M$ which is a ring isomorphism.

Proof. $N \otimes Q$ and $M \otimes Q$ are isomorphic as rings by Lemma 4.3. Therefore the product of $k$ elements in $M_{1} \otimes Q$ vanishes, and so the product of $k$ elements of $M_{1}$ vanishes. If the generators of $N$ are $x_{1}, x_{2}, \ldots, x_{m}$ of dimensions $n_{1} \leqq n_{2} \leqq \cdots \leqq n_{m}$, choose elements $u_{1}, u_{2}, \ldots, u_{m}$ in $M_{n_{1}}, M_{n_{2}}, \ldots, M_{n_{m}}$ such that $I_{n_{i}}\left(u_{i}\right)=x_{i}, 1 \leqq i \leqq m$. Set $J\left(x_{i}\right)=u_{i}, 1 \leqq i \leqq m$, which defines the required isomorphism.

Let $N$ be a truncated polynomial algebra of height $\geqq 3$ over $Q_{2}$. We may suppose that the height of $N$ is exactly 3 , for $M^{1}=\left(M_{1}\right)^{3}$ is an ideal which is a direct summand in $M$, and so, by Lemma 4.2 , we consider $M / M^{1}$.

The first significant result assisting in the classification of truncated polynomial algebras is the following

THEOREM 5.2. Let $N$ be a truncated polynomial algebra of height 3 on generators $x_{1}, x_{2}, \ldots, x_{m}$ of dimensions $n_{1} \leqq n_{2} \leqq \cdots \leqq n_{m}$. For each $r, 1 \leqq r \leqq m$, if $n_{s}$ and $n_{t}$ are chosen as large as possible with $n_{s}<2 n_{r}, n_{t}<n_{r}\left(n_{t}=0\right.$, if $\left.n_{r}=n_{1}\right)$, then

$$
\alpha \geqq \operatorname{Min}\left\{2 n_{r}-n_{s}, n_{r}-n_{t}\right\},
$$

where $\alpha=1$ if $n_{r}$ is odd and $\alpha=2+\nu_{2}\left(n_{r}\right)$ if $n_{r}$ is even.

Proof. Take a splitting which is a ring isomorphism, by Lemma 5.1.

Suppose that the theorem is false for some $r$, that is, if $n_{s}$ and $n_{t}$ are chosen as in the statement of the theorem, then $\alpha<\operatorname{Min}\left\{2 n_{r}-n_{s}, n_{r}-n_{t}\right\}$. Let $M^{1}$ be the ideal in $M$ generated by the elements $J\left(x_{u} x_{v}\right)$, where at least one of $n_{u}$ or $n_{v}$ is greater than or equal to $n_{r}$ and if one of $x_{u}$ or $x_{v}$ is $x_{r}$, the other is not and has dimension greater than or equal to $n_{r}$. Then $\psi^{k}\left(M^{1}\right) \subset M^{1}$ and $J\left(x_{r}^{2}\right) \notin M^{1}$. Lemma 4.6 implies that $S^{n} x_{r}=x_{r}^{2} \bmod 2$ in $M / M^{1}$. The required contradiction is now obtained by applying Theorem 3.3.

An immediate consequence of this theorem is the following well-known result.

THEOREM 5.3. Let $N$ be a truncated polynomial algebra of height $\geqq 3$ on one generator of dimension $n$. Then $n=1,2$ or 4 .

Theorem 5.2 is also used to prove the following result. 
THEOREM 5.4. Let $N$ be a truncated polynomial algebra of height $\geqq 3$ on two generators of dimensions $q$ and $r$. Then $(q, r)=(1,1),(1,2),(1,4),(2,2),(2,3),(2,4)$ or $(4,4)$.

Proof. Apply Theorem 5.2.

If $q=r$, then they have values 1,2 or 4 . We consider the three remaining possibilities (1) $q<r<2 q$, (2) $r=2 q$, (3) $2 q<r$, without loss of generality. Suppose that (a) $q$ and $r$ are both odd, (b) $q$ is odd and $r$ is even, (c) $q$ is even and $r$ is odd, and (d) $q$ and $r$ are both even.

(1)(a) $1 \geqq 2 q-r$ and $1 \geqq r-q$, which is impossible in this range.

(1)(b) $1 \geqq 2 q-r$ and $2+\nu_{2}(r) \geqq r-q$, but $r=2 q-1$ is impossible.

(1)(c) $2+\nu_{2}(q) \geqq 2 q-r$ and $1 \geqq r-q$ and so $r=q+1$ and $2+\nu_{2}(q) \geqq q-1$. The possibilities are $(2,3)$ and $(4,5)$.

(1)(d) $2+\nu_{2}(r) \geqq 2 q-r$ and $2+\nu_{2}(r) \geqq r-q$. Let $2^{s} \leqq q<2^{s+1}$ and so $2^{s}<r<2^{s+2}$; $4+\nu_{2}(q)+\nu_{2}(r) \geqq q$ implies that $5+2 s \geqq q \geqq 2^{s}$ and so $s=1,2$ or 3 . It is now routine to check that the only possibilities are $(4,6)$ and $(8,12)$.

(2) Clearly $q=1,2$ or 4 and so we obtain $(1,2),(2,4)$ or $(4,8)$.

(3) Once more $q=1,2$ or 4 . If $r$ is odd, $1 \geqq r-q$ and so $r=q+1$ which is impossible in this range. Therefore $r$ is even. If $q=1,2+\nu_{2}(r) \geqq r-1, r>2$ and we obtain $(1,4)$. If $q=2$ or 4 , then the conditions $4+\nu_{2}(r) \geqq r$ and $6+\nu_{2}(r) \geqq r$ hold respectively. Neither can be satisfied if $2 q>r$.

Therefore to complete the proof of Theorem 5.4, it remains to eliminate the cases $(4,5),(4,6),(4,8)$ and $(8,12)$. We eliminate these individually. The four arguments necessary involve four of the basic ideas necessary for the generalization to more then two generators.

We recall that since $J$ is a ring isomorphism, there is a Cartan formula over $Q_{2}$, by Corollary 4.5. The integer $k$ will always be an odd integer. Repeated use will be made of Corollary 2.11 and Lemma 2.12.

The case (4.5). Let the generators be $y_{4}$ and $y_{5}$. Then

$$
\left(1-k^{5}\right) S^{5} y_{5}=k^{4} T^{1}(k) S^{4} y_{5}+k^{3} T^{2}(k) S^{3} y_{5} \bmod 2^{5} .
$$

Now $S^{5} y_{5}=y_{5}^{2} \bmod 2$ by Lemma 4.6, and $T^{1}(k)=(1-k) S^{1}$. Taking $k=3 \bmod 4$, we deduce that $S^{4} y_{5}=y_{4} y_{5} \bmod 2$ and $S^{1} y_{4} y_{5}=y_{5}^{2} \bmod 2$. The second result implies that $S^{1} y_{4}=y_{5} \bmod 2$. Now

$$
\left(1-k^{6}\right) S^{6} y_{4}=k^{5} T^{1}(k) S^{5} y_{4}+k^{4} T^{2}(k) S^{4} y_{4}+k T^{5}(k) S^{1} y_{4} \quad \bmod 2^{6},
$$

which implies that

(5.1) $\left(1-k^{6}\right) S^{6} y_{4}=k^{5}(1-k) S^{1} S^{5} y_{4}+k^{4}\left(1-k^{2}\right) S^{2} S^{4} y_{4}+k^{5}(1-k) S^{1} S^{1} S^{4} y_{4} \bmod 2^{5}$.

Lemma 2.13 implies that $S^{6} y_{4}=0 \bmod 2$ and $S^{4} y_{4}=y_{4}^{2} \bmod 2$ by Lemma 4.6. Now if $k=3 \bmod 4$, (5.1) implies that $S^{1} S^{5} y_{4}=2 y_{5}^{2} \bmod 4$, which is immediately contradicted by taking $k=1 \bmod 4$ in this same equation. This is one result which can be proved more simply working over the mod 2 Steenrod algebra, as in [2]. 
The case (4.6). Let the generators be $y_{4}$ and $y_{6}$. Then $\left(1-k^{6}\right) S^{6} y_{6}=$ $k^{4} T^{2}(k) S^{4} y_{6} \bmod 2^{4}$, and so $S^{4} y_{6}=y_{4} y_{6} \bmod 2$ and $S^{2} y_{4} y_{6}=y_{6}^{2} \bmod 2$. The latter implies that $S^{2} y_{4}=y_{6} \bmod 2$, which we shall show is impossible. Let

$$
\Phi^{k}\left(y_{4}\right)=k^{4} y_{4}+a_{k} y_{6}+b_{k} y_{4}^{2}+c_{k} y_{4} y_{6}+d_{k} y_{6}^{2},
$$

and

$$
\Phi^{k}\left(y_{6}\right)=k^{6} y_{6}+e_{k} y_{4}^{2}+f_{k} y_{4} y_{6}+g_{k} y_{6}^{2} .
$$

We consider the equality $\Phi^{3} \Phi^{2}\left(y_{4}\right)=\Phi^{2} \Phi^{3}\left(y_{4}\right)$, which we may write out explicitly in the notation introduced above. In particular we consider dimensions 6 and 12 , and obtain the equations,

$$
\begin{gathered}
a_{3} 2^{4}\left(2^{2}-1\right)=a_{2} 3^{4}\left(3^{2}-1\right) \\
d_{3} 2^{4}\left(2^{8}-1\right)+c_{3} a_{2} 2^{6}+b_{3}\left(a_{2}\right)^{2}+a_{3} g_{2}=d_{2} 3^{4}\left(3^{8}-1\right)+c_{2} a_{3} 3^{6}+b_{2}\left(a_{3}\right)^{2}+a_{2} g_{3} .
\end{gathered}
$$

Since $S^{2} y_{4}=y_{6} \bmod 2, a_{2}$ is divisible by 4 but not by 8 , by Lemma 2.13 , and so $a_{3}$ is divisible by 2 but not by 4 . Lemma 4.8 implies that $d_{2}$ and $c_{2}$ are divisible by 2 , but $g_{2}$ is not and is nonzero. This is impossible as each term in the second equation above is divisible by 4 except $a_{3} g_{2}$.

The case (4.8). Let the generators be $y_{4}$ and $y_{8}$. Then

$$
\left(1-k^{8}\right) S^{8} y_{8}=k^{4} T^{4}(k) S^{4} y_{8} \bmod 2^{8}=k^{4}\left(1-k^{4}\right) S^{4} S^{4} y_{8} \quad \bmod 2^{8}
$$

but $S^{8} y_{8}=y_{8}^{2} \bmod 2$ and so $S^{4} S^{4} y_{8}=2 y_{8}^{2} \bmod 4$. Similarly,

$$
\left(1-k^{8}\right) S^{8} y_{4}=k^{4}\left(1-k^{4}\right) S^{4} S^{4} y_{4} \bmod 2^{8},
$$

but $S^{8} y_{4}=0 \bmod 2$ and so $S^{4} S^{4}=0 \bmod 4$.

Let $S^{4} y_{4}=\alpha y_{4}^{2}+\beta y_{8}$ and $S^{4} y_{8}=\gamma y_{4} y_{8}$, where $\alpha=1 \bmod 2$, by Lemma 4.6 and $\beta$ is even. Therefore $S^{4} S^{4} y_{8}=S^{4}\left(\gamma y_{4} y_{8}\right)=\beta \gamma y_{8}^{2}$, and $S^{4} S^{4} y_{4}=S^{4}\left(\alpha y_{4}^{2}+\beta y_{8}\right)=2 \alpha \beta y_{4} y_{8}$ $+\beta \gamma y_{4} y_{8}$. Hence $\beta \gamma=2 \bmod 4$ and $2 \alpha \beta+\beta \gamma=0 \bmod 4$, which is impossible since $\beta$ is even.

The case (8.12). Let the generators be $y_{8}$ and $y_{12}$. Then

$$
\left(1-k^{12}\right) S^{12} y_{12}=k^{8} T^{4}(k) S^{8} y_{12} \bmod 2^{8}=k^{8}\left(1-k^{4}\right) S^{4} S^{8} y_{12} \bmod 2^{8},
$$

and as $S^{12} y_{12}=y_{12}^{2} \bmod 2, S^{8} y_{12}=y_{8} y_{12} \bmod 2$ and $S^{4} y_{8} y_{12}=y_{12}^{2} \bmod 2$. The second result implies that $S^{4} y_{8}=y_{12} \bmod 2$. Now

$$
\left(1-k^{12}\right) S^{12} y_{8}=k^{8}\left(1-k^{4}\right) S^{4} S^{8} y_{8} \bmod 2^{8}=k^{8}\left(1-k^{4}\right) 2 y_{8} y_{12} \bmod 2^{6} .
$$

Therefore $S^{12} y_{8} \neq 0 \bmod 4$, which contradicts the fact that $2^{8} T^{12}(2) y_{8}=0 \bmod 2^{12}$, (Theorem 2.10). This completes the proof of Theorem 5.4.

When there are more than two generators, Theorem 5.2 by itself soon loses its efficiency, and so we now prove a number of lemmas without restriction upon the number of generators involved. The arguments can often be expressed in a number of different ways; at each stage we choose the one which seems to the author to be the shortest. 
Let the generators of the truncated polynomial algebra $N$ occur as usual in dimensions $n_{1} \leqq n_{2} \leqq \cdots \leqq n_{m}$.

Define the integer $t=t(n)=2^{s}$ to be the highest power of two which divides all the $n_{i}$.

LEMMA 5.5. $S^{t} S^{t}=0 \bmod 2$.

Proof. For odd $k,\left(1-k^{2 t}\right) S^{2 t}-k^{t}\left(1-k^{t}\right) S^{t} S^{t}=0 \bmod 2^{2 t}$. But $\nu_{2}\left(1-k_{t}^{2 t}\right)=3+s$ and $\nu_{2}\left(1-k^{t}\right)=2+s$, if $s>0$, or $\nu_{2}\left(1-k^{t}\right)=1$ if $s=0$, for suitable $k$. Therefore $S^{t} S^{t}=0 \bmod 2$.

The next lemma, which can be generalized, is closely connected with Theorem 2.1 of [18]. Once we have chosen generators for $N$, there is a natural base for $N$ over $Q_{2}$. We write I.E. for "independent elements" of this base and D.E. for decomposable elements. Also when we write $y_{n}$ for a generator, it is assumed that this generator has dimension $n$.

LEMMA 5.6. Let $y_{n}$ be a generator where $n=(2 k+1) 2^{s}, k>0$; then there is a generator $y_{n-t}$ such that $S^{t} y_{n-t}=y_{n}+$ I.E. $\bmod 2$.

Proof. Suppose that there is no generator $y_{n-t}$ with this property. For odd $k$,

$$
\left(1-k^{n}\right) S^{n} y_{n}=k^{n-t} T^{t}(k) S^{n-t} y_{n} \bmod 2^{2 t}=k^{n-t}\left(1-k^{t}\right) S^{t} S^{n-t} y_{n} \quad \bmod 2^{2 t} .
$$

Since $S^{n} y_{n}=y_{n}^{2} \bmod 2$ and $\nu_{2}\left(1-k^{n}\right)=\nu_{2}\left(1-k^{t}\right)$, it follows that there exists an element $z \in N_{2 n-t}$ with $S^{t} z=y_{n}^{2} \bmod 2$. This element $z$ must be a linear combination of generators of dimension $2 n-t$, products of generators of dimension $n-t$ with $y_{n}$ and other elements which when we apply $S^{t}$ can give no contribution to the coefficient of $y_{n}^{2} \bmod 2$. But our hypothesis on $y_{n}$ implies that no product of a generator of dimension $n-t$ with $y_{n}$ can give a nonzero contribution to $y_{n}^{2} \bmod 2$ under $S^{t}$. Thus there exists a generator $y_{2 n-t}$ with $S^{t} y_{2 n-t}=y_{n}^{2}+$ I.E. $\bmod 2$, and by suitable choice of generators of dimension $2 n-t$ it can be assumed to be the only generator with this property.

Now if there exists a generator $y_{2 n-2 t}$ of dimension $2 n-2 t$ with $S^{t} y_{2 n-2 t}=$ $y_{2 n-t}+$ I.E. $\bmod 2$, the coefficient of $y_{n}^{2}$ in $S^{t} S^{t} y_{2 n-2 t} \bmod 2$ is 1 , which contradicts Lemma 5.5. Hence $y_{2 n-t}$ is a generator possessing those properties which characterized $y_{n}$. Therefore there exists an infinite sequence of generators of dimensions $n, 2 n-t, 4 n-3 t, \ldots, 2^{q} n-\left(2^{q}-1\right) t, \ldots$ which contradicts the fact that $N$ is finitely generated. Thus our initial hypothesis was false and the lemma is proved.

COROLLARY 5.7. If there are $u$ generators of dimension $(2 k+1) 2^{s}, k>0$, there are at least $u$ generators of dimension $k \cdot 2^{s+1}$.

The proof is clear from that of Lemma 5.6.

Using Lemma 5.6, we now show how as a first step in classifying truncated polynomial algebras on more than two generators, it is possible to ignore odd dimensional generators. More precisely, we show how to find an ideal $M^{1}$ in $M$ 
which is a direct summand and is stable under the $\psi^{k}$, such that the graded ring corresponding to $M / M^{1}$ is just the subring of $N$ generated by the even-dimensional generators.

First consider a generator $y_{1}$, of dimension one. Choose a $J$ such that $\Phi_{J}^{k}\left(y_{1}\right)$ $=k y_{1}+a_{k} y_{1}^{2}$, for all $k$. This is possible, for by the technique described after Theorem 3.3 , by altering the splitting on $y_{1}$ we can ensure that $\Phi_{J}^{2}\left(y_{1}\right)=2 y_{1}+a_{2} y_{1}^{2}$. Further we may still assume that $J$ is a ring isomorphism by extending the splitting at each stage in the induction argument given in the discussion after Theorem 3.3 to be a ring isomorphism. The commuting condition then implies that $\Phi_{J}^{k}\left(y_{1}\right)=k y_{1}+a_{k} y_{1}^{2}$ for all $k$. Now the ideal in $M$ generated by $J\left(y_{1}\right)$ is a direct summand in $M$ which is stable under the $\psi^{k}$, so by Lemma 4.2 we may divide out by this ideal and in this manner neglect generators of dimension one.

Now consider a generator $y_{2 n+1}, n>0$. Lemma 5.4 implies the existence of a generator $y_{2 n}$ and these generators may be chosen such that $S^{1} y_{2 n}=y_{2 n+1}$. It follows that $\Phi^{k}\left(y_{2 n}\right)=k^{2 n} y_{2 n}+a_{k}^{(2 n)} y_{2 n+1}+$ higher-dimensional elements, where $\nu_{2}\left(a_{2}^{(2 n)}\right)=2 n-1$. Now $a_{k}^{(2 n)} 2^{2 n} 1=a_{2}^{(2 n)} k^{2 n}(k-1)$, so if $k=-1 \bmod 4$, then $a_{k}^{(2 n)}$ is odd and so it is a unit in $Q_{2}$. Therefore choose these generators and a splitting $J$ which is a ring isomorphism so that $\Phi_{J}^{-1}\left(y_{2 n}\right)=y_{2 n}+y_{2 n+1}$. Since $\Phi^{-1} \Phi^{-1}=\Phi^{1}$, the identity, we have that $\Phi^{-1}\left(y_{2 n+1}\right)=-y_{2 n+1}$. Let this process be performed simultaneously for each odd-dimensional generator with dimension greater than one and let $M^{1}$ be the ideal in $M$ generated by the $J\left(y_{i}\right)$, where $y_{i}$ runs over all the odddimensional generators as above.

LEMMA 5.8. $\psi^{k}\left(M^{1}\right) \subset M^{1}$, for each $k$.

Proof. $\Phi^{k} \Phi^{-1}\left(y_{2 n+1}\right)=\Phi^{-1} \Phi^{k}\left(y_{2 n+1}\right)$, and so $\Phi^{k}\left(y_{2 n+1}\right)=-\Phi^{-1} \Phi^{k}\left(y_{2 n+1}\right)$. It follows that when $\Phi^{k}\left(y_{2 n+1}\right)$ is expressed in terms of the base, it is independent of even-dimensional generators and of products of even-dimensional generators. The result follows.

Therefore, by Lemma 4.2 , consider $M / M^{1}$, whose corresponding graded ring is just the subring of $N$ generated by the even-dimensional generators. Until Theorem 5.15, it is assumed that all the generators of $N$ occur in even dimensions. Once and for all, certain choices of generators are made. Whenever there is a generator $y_{4 n+2}, n>0$, there is a generator $y_{4 n}$, by Lemma 5.6, and it is assumed that the generators are chosen so that $S^{2} y_{4 n}=y_{4 n+2}$. Also $S^{2} y_{4 n}^{\prime}$ is independent of $y_{4 n+2}$ for any other generator of dimension $4 n$.

A simple verification based on Corollary 2.11 implies that $S^{2} S^{2}=S^{3} S^{1} \bmod 2$ and so $S^{2} y_{4 n+2}=0 \bmod 2$. If in addition $S^{2} y_{4 n+2}=0 \bmod 4$ or $S^{2} y_{4 n+2}=$ D. E. $\bmod 4$, by using the technique described after Theorem 3.3, it can be assumed that $S^{2} y_{4 n+2}$ $=0$ or $S^{2} y_{4 n+2}=$ D.E. respectively.

All the above choices are compatible with the splitting remaining a ring isomorphism. 
LEMMA 5.9. $t(N)=2^{s} \leqq 4$.

Proof. Let $y_{m}$ be a generator with $m$ not divisible by $2^{s+1}$. Theorem 5.2 implies that $2+s \geqq \operatorname{Min}\left\{2 m-m_{1}, m-m_{2}\right\}$, where $m_{1}$ and $m_{2}$ are given by that proposition. But $\nu_{2}\left(2 m-m_{1}\right)$ and $\nu_{2}\left(m-m_{2}\right)$ are greater than or equal to $s$. Therefore $2 m-m_{1}$ $\geqq 2^{s}, m-m_{2} \geqq 2^{s}$, and so $2+s \geqq 2^{s}$, which implies that $s \leqq 2$.

LEMMA 5.10. Let $y_{8 n+4}, n>0$, be a generator of highest possible dimension not divisible by 8 . Then there is a generator of dimension greater than $8 n-4$ not divisible by 4 .

Proof. Suppose that the conclusion is false and there are no generators of dimension $8 n-2$ or $8 n+2$. As usual for odd $k$,

$$
\left(1-k^{8 n+4}\right) S^{8 n+4} y_{8 n+4}=k^{8 n+2} T^{2}(k) S^{8 n+2} y_{8 n+4}+k^{8 n} T^{4}(k) S^{8 n} y_{8 n+4} \bmod 2^{6} .
$$

For dimensional reasons $S^{2} S^{8 n+2} y_{8 n+4}$ and $S^{2} S^{2} S^{8 n} y_{8 n+4}$ are independent of $y_{8 n+4}^{2}$, and since $S^{8 n+4} y_{8 n+4}=y_{8 n+4}^{2} \bmod 2$, there are generators of dimension $8 n$ such that $S^{4} y_{8 n}=y_{8 n+4}+$ I.E. $\bmod 2$. Choose the generators in dimension $8 n$ so that $y_{8 n}$ is the only generator with this property and let $S^{4} y_{8 n}=y_{8 n+4}$.

We shall obtain two contradictory results on the coefficient of $y_{8 n} y_{8 n+4}$ in $S^{4} S^{8 n} y_{8 n} \bmod 4$.

First it is shown that this coefficient is zero. Theorem 2.10 implies that

$$
2^{8 n}\left\{\left(1-2^{8 n+4}\right) S^{8 n+4} y_{8 n}+2^{2}\left(1-2^{8 n+2}\right) S^{8 n+2} Q^{2} y_{8 n}\right\}=0 \bmod 2^{8 n+4},
$$

and so $S^{8 n+4} y_{8 n}=0 \bmod 4$. Also, for odd $k$,

$$
\left(1-k^{8 n+4}\right) S^{8 n+4} y_{8 n}=k^{8 n+2} T^{2}(k) S^{8 n+2} y_{8 n}+k^{8 n} T^{4}(k) S^{8 n} y_{8 n} \bmod 2^{6} .
$$

But $S^{2} S^{8 n+2} y_{8 n}$ and $S^{2} S^{2} S^{8 n} y_{8 n}$ are independent of $y_{8 n} y_{8 n+4}$ for dimensional reasons, and so the coefficient of $y_{8 n} y_{8 n+4}$ in $S^{4} S^{8 n} y_{8 n} \bmod 4$ is zero.

Using an alternative argument, we show that this coefficient must be 2 . Since there are no odd-dimensional elements, it can be verified from Corollary 2.11 that $S^{4} S^{4}=S^{6} S^{2} \bmod 2$. Therefore if there exists a generator $y_{16 n}$, the coefficient of $y_{8 n} y_{8 n+4}$ in $S^{4} y_{16 n} \bmod 2$ is zero, since $S^{4} S^{4} y_{16 n}=S^{6} S^{2} y_{16 n} \bmod 2$ and the coefficient of $y_{8 n+4}^{2}$ on the right-hand side is zero.

Let $S^{8 n} y_{8 n}=\alpha y_{8 n}^{2}+\beta y_{16 n}+\gamma y_{8 n-4} y_{8 n+4}+$ I.E., where $\alpha$ is odd, $\beta, \gamma$ are even and $y_{8 n-4}$ is a typical generator of dimension $8 n-4$. Then the coefficient of $y_{8 n}$ in $S^{4} y_{8 n-4} \bmod 2$ is zero, for $S^{4} S^{4} y_{8 n-4}=S^{6} S^{2} y_{8 n-4} \bmod 2$ and there are no generators of dimension $8 n-2$. Thus the coefficient of $y_{8 n} y_{8 n+4}$ in $S^{4} S^{8 n} y_{8 n} \bmod 4$ is $2 \alpha=2$. This completes the proof of the lemma.

LEMMA 5.11. Let $N$ have a generator of dimension $4 n+2, n>0$. Then

(a) $N$ has generators of dimensions $4 n-2$ or $8 n-2$,

(b) $N$ has generators of dimensions $4 n-2$ or $8 n$. 
Proof of (b). $\Phi^{k}\left(y_{4 n}\right)=k^{4 n} y_{4 n}+a_{k}^{(4 n)} y_{4 n+2}+$ higher-dimensional elements. But $a_{k}^{(4 n)} 2^{4 n}\left(1-2^{2}\right)=a_{2}^{(4 n)} k^{4 n}\left(1-k^{2}\right)$ and since $\nu_{2}\left(a_{2}^{(4 n)}\right)=4 n-2$, it follows that $\nu_{2}\left(a_{5}^{(4 n)}\right)=1$.

The argument we now use is of a type which will occur repeatedly and so we give it in some detail. Let $\zeta_{k}^{(t)}$ be the coefficient of $y_{4 n+2}^{2}$ in $\Phi^{k}\left(y_{t}\right)$. Consider the coefficient of $y_{4 n+2}^{2}$ in $\Phi^{2} \Phi^{5}\left(y_{4 n}\right)=\Phi^{5} \Phi^{2}\left(y_{4 n}\right)$ modulo 4 . The coefficient of $y_{4 n+2}^{2}$ in $\Phi^{2} \Phi^{5}\left(y_{4 n}\right) \bmod 4$ is $5^{4 n} \zeta_{2}^{(4 n)}+a_{5}^{(4 n)} \zeta_{2}^{(4 n+2)}$. There is no contribution from the higher-dimensional elements in the expression for $\Phi^{5}\left(y_{4 n}\right)$ modulo 4 for dimensional reasons, using Lemma 2.13(b). Now consider $\Phi^{5} \Phi^{2}\left(y_{4 n}\right) \bmod 4$. As we are considering coefficients in dimension $8 n+4$, it is sufficient to consider $\Phi^{2}\left(y_{4 n}\right)$ in dimensions $8 n, 8 n+2$, and $8 n+4$. Let

$$
\begin{aligned}
& \Phi^{2}\left(y_{4 n}\right)=\alpha_{2}^{(4 n)} y_{4 n}^{2}+\beta_{2}^{(4 n)} y_{8 n}+\gamma_{2}^{(4 n)} y_{4 n-2} y_{4 n+2} \\
&+ \delta_{2}^{(4 n)} y_{4 n-4} y_{4 n+4}+\cdots+w_{2}+\cdots+\zeta_{2}^{(4 n)} y_{4 n+2}^{2},
\end{aligned}
$$

where the first group of terms are typical elements of dimension $8 n$ and $w_{2}$ lies in dimension $8 n+2$. Now $w_{2}$ is divisible by 2 and so the coefficient of $y_{4 n+2}^{2}$ in $\Phi^{5}\left(w_{2}\right)$ is divisible by 4 , by the same argument as above which implied that $\nu_{2}\left(a_{5}^{(4 n)}\right)$ is not zero. The contribution from $\alpha_{2}^{(4 n)} y_{4 n}^{2}$ is likewise zero $\bmod 4$, since $a_{5}^{(4 n)}$ is divisible by 2 and $\Phi^{5}$ is a ring homomorphism. There is no contribution from $y_{4 n-4} y_{4 n+4}$, etc., for dimensional reasons. Therefore if there are no generators of dimension $4 n-2$ or $8 n$, the coefficient of $y_{4 n+2}^{2}$ in $\Phi^{5} \Phi^{2}\left(y_{4 n}\right) \bmod 4$ is $\zeta_{2}^{(4 n)} 5^{8 n+4}$. Therefore $\zeta_{2}^{(4 n)} 5^{8 n+4}=5^{4 n} \zeta_{2}^{(4 n)}+a_{5}^{(4 n)} \zeta_{2}^{(4 n+2)} \bmod 4$ or $5^{4 n}\left(1-5^{4 n+4}\right) \zeta_{2}^{(4 n)}=a_{5}^{(4 n)} \zeta_{2}^{(4 n+2)} \bmod 4$ and so $a_{5}^{(4 n)} \zeta_{2}^{(4 n+2)}=0 \bmod 4$. This is false, as $\zeta_{2}^{(4 n+2)}$ is odd, by Lemma 4.8 , and we have shown above that $a_{5}^{(4 n)}$ is not divisible by 4 . Therefore there is a generator of dimension $4 n-2$ or a generator of dimension $8 n$.

This argument is a direct generalization of the argument that eliminated the special case $(4,6)$ in Theorem 5.4. After some practice, such arguments can be performed mentally and so we abbreviate the above to

Considering $y_{4 n+2}^{2}$ in $F\left(2,5 ; y_{4 n}\right) \bmod 4$, where $S^{2} y_{4 n}=y_{4 n+2}$ (and hence $\nu_{2}\left(a_{5}^{(4 n)}\right)=1$ ), deduce the existence of a generator of dimension $4 n-2$ or $8 n$,

where "considering $y_{4 n+2}^{2}$ in $F\left(2,5 ; y_{4 n}\right)$ mod 4 " means, considering the coefficient of $y_{4 n+2}^{2}$ in the equation $\Phi^{2} \Phi^{5}\left(y_{4 n}\right)=\Phi^{5} \Phi^{2}\left(y_{4 n}\right) \bmod 4$.

Proof of Lemma 5.11 (a). Considering $y_{4 n} y_{4 n+2}$ in $F\left(2,5 ; y_{4 n}\right) \bmod 8$, where $S^{2} y_{4 n}=y_{4 n+2}$, deduce the existence of a generator of dimension $4 n-2$ or $8 n-2$.

The next theorem is the classification theorem, assuming that $N$ has only evendimensional generators. The proof will involve the elimination of several particular cases. These will be considered separately at the end of this section. We shall write $\left[n_{1}, n_{2}, \ldots\right]$, as distinct from $\left(n_{1}, n_{2}, \ldots\right)$ to include the possibility of having more than one generator in a given dimension. 
THEOREM 5.12. Let the generators of $N$ be even dimensional and not more than five in number; then the set of dimensions of these generators is a union of sets taken from (2), (4), $(2,4,6),(2,4,6,8)$ and $(2,4,6,8,10)$.

This is the main part of the proof of Theorem 1.1, and the proof will take most of the remainder of this section. We assume the hypotheses of Theorem 5.12 until Theorem 5.15 is reached.

Lemma 5.13. Let $N$ have a generator of dimension greater than 14. Then $N$ has a generator whose dimension is greater than 8 and is not divisible by 4 .

Proof. Suppose first that the generator of highest dimension occurs in dimension 16. Theorem 5.2 implies that there is a generator of dimension greater than 8 . If there are no generators of dimension 10 or 14 , there must be a generator of dimension 12 and again applying Theorem 5.2, there must be a generator of dimension 8. Lemma 5.10 implies that there is a generator of dimension 6 and so, by Lemma 5.6, there is a generator of dimension 4 . Therefore it is necessary to eliminate the special case $(4,6,8,12,16)$.

Now suppose that there exists a generator of dimension greater than 16. If $m$ is any integer with $\nu_{2}(m)=\alpha>2$, it is easy to see that there are no integers $n$ in the range $m-2-\alpha \leqq n<m$ with $\nu_{2}(n) \geqq \nu_{2}(m)$. This implies by Theorem 5.2 that if there is a generator of dimension $>16$, then there is a generator of dimension not divisible by 8 and $>16$. For if $y_{m}$ has dimension $>16$ and $\nu_{2}(m)>2$, Theorem 5.2 implies that there exists a generator of dimension $n$ with $\nu_{2}(n)<\nu_{2}(m)$. But 16 is less than $n-2-\nu_{2}(n)$, for $2^{r}$ is less than $2^{r+1}-2-r$ if $r \geqq 3$, and so $n>16$. After applying Theorem 5.2 a finite number of times, we deduce the existence of a generator of dimension $>16$ and not divisible by 8 . If it is not divisible by 4 we are done; otherwise we apply Lemma 5.10 to complete the proof.

LEMMA 5.14. Excluding the case (2, 4, 6, 8, 10), the highest-dimensional generator of $N$ with dimension not divisible by 4 occurs in dimension 2 or 6 .

Proof. First suppose that the generator of highest dimension not divisible by 4 occurs in dimension $4 n+2$, with $n>3$. Lemma 5.6 implies the existence of a generator of dimension $4 n$ and Lemma 5.11(a) implies one of dimension $4 n-2$. Again apply Lemma 5.6 to deduce the existence of a generator of dimension $4 n-4$ and Lemma 5.11(a) to show that there is a generator of dimension $4 n-6$. Finally Lemma 5.6 implies the existence of a generator of dimension $4 n-8$. There are now at least six generators and so this case is disregarded.

Therefore suppose that 14 is the highest dimension which occurs and is not divisible by 4 . As above, deduce the existence of generators of dimensions 12, 10 and 8. Now Lemma 5.11 implies that either there is a generator of dimension 6 or a generator of dimension 16. If there is a generator of dimension 6 , then there is a generator of dimension 4 and there are at least six generators. Therefore the 
only possibility with which we are concerned is $(16,14,12,10,8)$, which will be considered at the end of the section.

Next suppose that the generator of highest dimension not divisible by 4 occurs in dimension 10. It follows as above that there exist generators in dimensions 8, 6 and 4. Using Theorem 5.2, it is easy to check that there are just four possible sets of dimensions, namely $[4,6,8,10],(2,4,6,8,10)(4,6,8,10,12)$ or $(4,6,8,10,16)$. All except $(2,4,6,8,10)$ will be eliminated.

This completes the proof of the lemma.

Proof of Theorem 5.12. If all generators lie in a single dimension, it follows from Theorem 5.2 that this dimension is 2 or 4 .

If all generators lie in just two dimensions, then the first part of the proof of Theorem 5.4 implies that these dimensions are $[2,4],[4,6],[4,8]$ or $[8,12]$ and Lemmas 5.11 and 5.10 imply that $[4,6]$ and $[8,12]$ are impossible. It remains to eliminate $[4,8]$.

Suppose that there are three distinct dimensions. Then Theorem 5.14 implies that 6 or 2 is the highest possible dimension not divisible by 4 . Therefore by Lemma 5.13, the generators all must lie in dimensions $2,4,6,8$ or 12 . Using Lemma 5.10, Lemma 5.11 and Lemma 5.6, there are just three possibilities $[2,4,6],[2,4,8]$ or $[4,6,8]$. Now Corollary 5.7 implies that there can be at most two generators of dimension 6 ; therefore it is only necessary to eliminate $(2,4,4,6,6),[2,4,8]$ and $[4,6,8]$.

Suppose that there are four distinct dimensions. The arguments used above reduce this to the consideration of $[2,4,6,8]$ and $[4,6,8,12]$. In the first case there can only be one generator of dimension 6 , by Corollary 5.7, and so we need just eliminate $(2,4,6,8,8)$. The second case will be shown to be impossible.

Finally we come to the case of five distinct dimensions, which by Lemmas 5.13 and 5.14 must be $(2,4,6,8,10)$ or $(2,4,6,8,12)$. The second case will be eliminated.

Therefore, except for the elimination of several particular cases, the proof of Theorem 5.12 is completed.

It is no longer assumed that all the generators have even dimensions.

THEOREM 5.15. Let $N$ have not more than five generators; then the set of dimensions of these generators is a union of sets taken from (1), (2), (4), (2, 3), (2, 4, 6), (2, 3, 4, 5) $(2,4,6,8),(2,3,4,5,6)$ and $(2,4,6,8,10)$.

Take a splitting of the type constructed after Corollary 5.7. For the reasons explained there, we may ignore any generators of dimension 1 . Also notice that for such a splitting, $S^{2 n+1} y_{2 m+1}=0$, for all $m$ and $n$.

LEMMA 5.16. Let $N$ have a generator of dimension $4 n+1$. Then $N$ has a generator of dimension $4 n-1$ or $8 n-1$. 
Proof. From the construction of the splitting, it follows that there is a unique generator $y_{4 n}$ such that $S^{1} y_{4 n}=y_{4 n+1}$. Let

$$
\Phi^{k}\left(y_{4 n}\right)=k^{4 n} y_{4 n}+\alpha_{k}^{(4 n)} y_{4 n+1}+\text { higher-dimensional elements. }
$$

Then $\nu_{2}\left(\alpha_{5}^{(4 n)}\right)=1$.

If there is a generator of dimension $4 n-2$, but no generator of dimension $4 n-1$, let

$$
\Phi^{k}\left(y_{4 n-2}\right)=k^{4 n-2} y_{4 n-2}+a_{k} y_{4 n}+b_{k} y_{4 n+1}+\text { I.E. }
$$

Consider $y_{4 n+1}$ in $F\left(2, k ; y_{4 n-2}\right)$ to obtain

$$
2^{4 n-2}\left(2^{3}-1\right) b_{k}+a_{k} \alpha_{2}=k^{4 n-2}\left(k^{3}-1\right) b_{2}+a_{2} \alpha_{k} .
$$

Now $\nu_{2}\left(a_{2}\right)>4 n-4$, since $S^{2} y_{4 n-2}=0 \bmod 2$ and so $\nu_{2}\left(a_{5}\right)=\nu_{2}\left(a_{3}\right) \geqq 2$. Putting $k$ alternately equal to 3 and 5 in (5.2), deduce that $b_{5}$ is even. Considering $y_{4 n+1}^{2}$ in $F\left(2,5 ; y_{4 n}\right) \bmod 4$, deduce the existence of a generator of dimension $8 n-1$, assuming that there is no generator of dimension $4 n-1$, whether or not there is a generator of dimension $4 n-2$.

Proof of Theorem 5.15. By the remarks following Corollary 5.7, the evendimensional generators are as in Theorem 5.12. Also if there is a generator of dimension $2 n+1, n>0$, there is a generator of dimension $2 n$, by Lemma 5.6.

Suppose that $N$ has a generator of odd dimension greater than one. If the evendimensional generators occur in just one dimension, it must be 2 or 4 . It follows from Lemma 5.16 that the only possibility is $[2,3]$, and by Corollary 5.7 , we have $(2,3),(2,2,3,3)$ or $(2,2,2,3,3)$.

If the even-dimensional generators occur in just two dimensions, they are 2 and 4. Again using Lemma 5.16, the possibilities are [2, 3, 4] or $[2,3,4,5]$ where in the first case there are at least as many generators of dimension 2 as there are of dimension 3 and in the second case there can only be more than one generator in dimension 2 or 4 .

If even generators occur in three dimensions, they are $[2,4,6]$. The possibilities are $[2,3,4,6],[2,4,6,7]$ or $(2,3,4,5,6)$. The first two cases will be considered later.

If even-dimensional generators occur in four dimensions, they are $[2,4,6,8]$. The possibilities are $(2,3,4,6,8)$ or $(2,4,6,7,8)$, once more using Lemma 5.16.

Therefore to complete the proof of Theorem 5.15, it remains to consider the special cases above and those remaining from Theorem 5.12, which are $(8,10,12,14,16),(4,6,8,12,16),(4,6,8,10,16),(4,6,8,10,12),[4,6,8,12]$, $[4,6,8,10],[4,6,8],[2,4,8],[4,8],(2,3,4,6,8),(2,4,6,7,8),[2,3,4,6]$ and $[2,4,6,7]$. It is also necessary to consider $[2,4,6,8]$ and $[2,4,6]$. The details will be given in rather greater length than is usually the case, as the techniques used are not standard and these exceptional cases tend to correspond to those examples which cannot easily be eliminated working with the Steenrod algebra. We remind 
the reader of the choices of generators that are made after Lemma 5.8. Also, when there are no odd-dimensional generators, $\Phi^{k}\left(y_{2 t}\right)=k^{2 t} \sum_{i \geqq 0} R^{2 i}(k) y_{2 t}$, for all $k$. It follows from the definition of $R^{2 i}(2)$, that $2^{2(t-i)} R^{2 i}(2) y_{2 t}=S^{2 i} y_{2 t} \bmod 4$; in particular $R^{2 t}(2) y_{2 t}=S^{2 t} y_{2 t} \bmod 4$.

$(8,10,12,14,16)$. Considering $y_{14}^{2}$ in $F\left(2,5 ; y_{12}\right) \bmod 4$, deduce that $R^{4}(5) y_{10}$ $=y_{14} \bmod 2$. Considering $y_{10} y_{14}$ in $F\left(2,5 ; y_{10}\right) \bmod 4$, obtain a contradiction.

$(4,6,8,12,16)$. Considering $y_{6}^{2}$ in $F\left(2,5 ; y_{4}\right) \bmod 64$, deduce that $S^{4} y_{4}=\alpha y_{4}^{2}$ $+\beta y_{8}$, where $\nu_{2}(\alpha)=0$ and $\nu_{2}(\beta)=1$. Considering $y_{12}$ in $F\left(2,5 ; y_{4}\right) \bmod 4$, deduce that $R^{4}(5)=$ D.E. $\bmod 2$. Considering $y_{12}^{2}$ in $F\left(2,5 ; y_{12}\right)$ mod 16 , obtain a contradiction.

$(4,6,8,10,16)$. The argument below which applies to $[4,6,8,10]$ will work here.

$(4,6,8,10,12)$. This is the most difficult special case with which we are concerned; see the remark after Theorem 3 in [13].

Considering $y_{10}^{2}$ in $F\left(2,5 ; y_{8}\right) \bmod 4$, deduce that $R^{4}(5) y_{6}=y_{10}+$ D.E. $\bmod 2$.

Considering $y_{6} y_{10}$ in $F\left(2,5 ; y_{6}\right) \bmod 4$, deduce that $S^{6} y_{6}=2 y_{12}+$ D.E. $\bmod 4$.

Considering $y_{12}$ in $F\left(2,5 ; y_{6}\right) \bmod 32$, deduce that $S^{4} y_{6}=y_{10}+$ D.E. $\bmod 2$ and $S^{2} y_{10}=2 y_{12}+$ D.E. $\bmod 4$.

Considering $y_{10}$ in $F\left(2,5 ; y_{6}\right) \bmod 32$, and using the facts that $S^{2} y_{8}=y_{10}$, $R^{4}(5) y_{6}=y_{10}+$ D.E. $\bmod 2$ and $S^{4} y_{6}=y_{10}+$ D.E. $\bmod 2$, deduce that $S^{2} y_{6}=$ D.E. $\bmod 4$, and so $S^{2} y_{6}=$ D.E.

Considering $y_{6}^{2}$ in $F\left(2,5 ; y_{4}\right) \bmod 4$, deduce that $S^{4} y_{4}=2 y_{8}+$ D.E. $\bmod 4$.

Considering $y_{12}$ in $F\left(2,5 ; y_{4}\right) \bmod 4$, deduce that $R^{4}(5) y_{8}=$ D.E. $\bmod 2$.

Considering $y_{10}^{2}$ in $F\left(2,5 ; y_{10}\right) \bmod 16$, deduce that $S^{8} y_{10}=y_{8} y_{10}+$ I.E. $\bmod 2$.

Considering $y_{8} y_{12}$ in $F\left(2,5 ; y_{10}\right) \bmod 32$, deduce that $S^{10} y_{10}=2 y_{8} y_{12}+$ I.E. $\bmod 4$.

Considering $y_{12}^{2}$ in $F\left(2,5 ; y_{10}\right) \bmod 8$, deduce that if $R^{4}(5) y_{8}=2 y_{12}+$ D.E. $\bmod 4$, then $R^{6}(5) y_{6}=$ D.E. $\bmod 2$. Now if $R^{4}(5) y_{8} \neq 2 y_{12}+$ D.E. $\bmod 4$, by defining a new splitting such that $K\left(y_{10}\right)=J\left(y_{10}+y_{12}\right), K\left(y_{i}\right)=J\left(y_{i}\right)$ otherwise, we ensure that $R^{4}(5) y_{8}=2 y_{12} \bmod 4$.

Considering $y_{6} y_{12}$ in $F\left(2,5 ; y_{8}\right) \bmod 8$, deduce that the coefficient of $y_{4} y_{12}$ in $\Phi^{2}\left(y_{8}\right)$ is divisible by 4 . Finally a contradiction is obtained by considering $y_{12}^{2}$ in $F\left(2,5 ; y_{8}\right) \bmod 4$.

$[4,6,8,12]$. As in the case $[4,6,8]$, deduce that $S^{2} y_{6}=$ D.E., and so $R^{4}(5) y_{4}$ $=$ D.E. $\bmod 2$.

Considering $y_{12}^{2}$ in $F\left(2,5 ; y_{12}\right) \bmod 16$, for some $y_{12}$, deduce that $R^{4}(5) y_{8}$ $=y_{12}+$ I.E. $\bmod 2$ for some $y_{8}$. Contradict this by considering $y_{8} y_{12}$ in $F\left(2,5 ; y_{8}\right)$ $\bmod 4$.

$[4,6,8,10]$. Corollary 5.7 implies that there can be only one generator in the dimensions 6 and 10 . Considering $y_{10}^{2}$ in $F\left(2,5 ; y_{8}\right) \bmod 4$, deduce that there is a generator $y_{6}$ with $R^{4}(5) y_{6}=y_{10}+$ D.E. $\bmod 2$. Considering $y_{6} y_{10}$ in $F\left(2,5 ; y_{6}\right)$ $\bmod 4$, obtain a contradiction.

$[4,6,8]$. It is first shown that $S^{2} y_{6}=$ D.E., for suppose not, and let $S^{2} y_{6}=2 y_{8}$ + I.E. $\bmod 4$, for some generator $y_{8}$. 
Considering $y_{8}$ in $F\left(2,5 ; y_{4}\right) \bmod 32$, deduce that $R^{4}(5) y_{4}=y_{8}+$ I.E. $\bmod 2$.

Considering $y_{6}^{2}$ in $F\left(2,5 ; y_{6}\right) \bmod 16$, deduce that $S^{4} y_{6}=y_{4} y_{6}+$ I.E. $\bmod 2$.

Considering $y_{6} y_{8}$ in $F\left(2,5 ; y_{6}\right) \bmod 8$, deduce that $S^{6} y_{6}=2 y_{4} y_{6}+$ I.E. $\bmod 4$.

Considering $y_{8}^{2}$ in $F\left(2,5 ; y_{6}\right) \bmod 8$, obtain a contradiction. Thus $S^{2} y_{6}=$ D.E.

Considering $y_{8}^{2}$ in $F\left(2,5 ; y_{8}\right) \bmod 64$, deduce that $S^{4} y_{8}=y_{4} y_{8}+$ I.E. $\bmod 2$ and $S^{4} y_{4}=2 y_{8}+$ I.E. $\bmod 4$, for some generator $y_{8}$.

Hence $R^{4}(5) y_{8}=y_{4} y_{8}+$ I.E. $\bmod 2$, and so for a suitable splitting the coefficient of $y_{8}^{2}$ in $R^{8}(5) y_{8} \bmod 2$ is zero, for if this is not true originally set $K\left(y_{4}\right)=J\left(y_{4}+y_{8}\right)$, $K\left(y_{i}\right)=J\left(y_{i}\right)$ otherwise.

Considering $y_{8}^{2}$ in $F\left(2,5 ; y_{4}\right) \bmod 4$, obtain a contradiction.

$[2,4,8]$. Corollary 2.11 and Lemma 2.12 , imply that, for odd $k$,

$$
\left(1-k^{8}\right) S^{8} y_{8}=k^{4}\left(1-k^{4}\right) S^{4} S^{4} y_{8} \bmod 2^{6} .
$$

Since $S^{8} y_{8}=y_{8}^{2} \bmod 2$, it follows that $S^{4} S^{4} y_{8}=2 y_{8}^{2} \bmod 4$. Now let $S^{4} y_{8}=\alpha y_{4}^{\prime} y_{8}^{\prime}$ $+\beta y_{4}^{\prime \prime} y_{8}^{\prime \prime}+\cdots$. Since $S^{4} S^{4} y_{8}=2 y_{8}^{2} \bmod 4$, deduce that one of $y_{8}^{\prime}, y_{8}^{\prime \prime}$, is $y_{8}, y_{8}^{\prime}$ say, and $S^{4} y_{4}^{\prime}=2 y_{8}+$ D.E. $\bmod 4$, where $\alpha=1 \bmod 2$. Choose generators so that $y_{4}^{\prime}$ is the only generator with this property and write $y_{4}$ for $y_{4}^{\prime}$. It will still be true that $\alpha=1 \bmod 2$. Now

$$
\left(1-k^{8}\right) S^{8} y_{4}=k^{6} T^{2}(k) S^{6} y_{4}+T^{4}(k) S^{4} y_{4} \bmod 2^{6} .
$$

It follows that $S^{4} S^{4} y_{4}=0 \bmod 4$, since $S^{8} y_{4}=0 \bmod 2$ and $S^{2} S^{6} y_{4}=0 \bmod 8$, using Theorem 2.10 with $k=2$.

Let $S^{4} y_{4}=\zeta y_{4}^{2}+\eta y_{4}^{\prime \prime 2}+\cdots+2 \theta y_{8}+4 \xi y_{8}^{\prime}$, where $\theta=1 \bmod 2$ and $\zeta=1 \bmod 2$. Then $S^{4} S^{4} y_{4}=2 \zeta \theta y_{4} y_{8}+$ I.E. $\bmod 4$, which contradicts the earlier fact that $S^{4} S^{4} y_{4}$ $=0 \bmod 4$.

$[4,8]$. This is a simplified version of $[2,4,8]$.

To complete the proof of Theorem 5.12 it remains to consider $[2,4,6,8]$ and $[2,4,6]$.

$[2,4,6,8]$. It is necessary to show that there can only be more than one generator in dimension 2 or 4 . There can only be one generator in dimension 6 , by Corollary 5.7; therefore consider $(2,4,6,8,8)$. As before, see e.g. $(4,6,8)$, one proves that $S^{2} y_{6}=$ D.E. $\bmod 4$ and therefore $S^{2} y_{6}=$ D.E. It follows that $R^{4}(5) y_{4}=2 y_{8}+$ I.E. mod 4. By suitable choice of generators, we may suppose that the coefficient of $y_{8}^{\prime}$, the other generator of dimension 8 , in $R^{4}(5) y_{4} \bmod 4$ is zero.

Consider $y_{8}^{\prime 2}$ in $F\left(2,5 ; y_{8}^{\prime}\right) \bmod 64$, to obtain a contradiction.

$[2,4,6]$. Again applying Corollary 5.7 , it is only necessary to eliminate $(2,4,4,6,6)$. Let $S^{2} y_{4}=y_{6}$ and by considering $y_{6}^{2}$ in $F\left(2,5 ; y_{4}\right) \bmod 4$ deduce that $R^{4}(5) y_{2}=y_{6}+$ I.E. $\bmod 2$. There is just one generator of dimension 2 , but two generators of dimension 6 , which is clearly impossible.

This completes the proof of Theorem 5.12.

Now consider the particular cases when there is an odd-dimensional generator. 
$[2,3,4,6]$. Consider $y_{6}^{2}$ in $F\left(2,5 ; y_{4}\right) \bmod 4$, and deduce that $S^{4} y_{4}=\alpha y_{4}^{2}+2 y_{2} y_{6}$ $\bmod 4$, where $\alpha=1 \bmod 2$.

Considering $F\left(2,-1 ; y_{4}\right)$ mod 8 on $y_{3} y_{6}$, obtain the necessary contradiction.

$(2,3,4,6,7)$. The argument of $[2,3,4,6]$ eliminates this case.

$(2,3,4,6,8)$. Here the tactic is a little different; we reduce this case to $(2,3,4,6)$.

Provided that $R^{4}(5) y_{2}=y_{6}+$ D.E. $\bmod 2$, it is possible to find $\alpha, \beta$ and $\gamma$ in $Q_{2}$, such that $\Phi^{5}\left(y_{8}+\alpha y_{4}^{2}+\beta y_{2} y_{6}+\gamma y_{6}^{2}\right)$ is independent of $y_{4} y_{6}$ and $y_{6}^{2}$, and therefore $\Phi^{k}\left(y_{8}+\alpha y_{4}^{2}+\beta y_{2} y_{6}+\gamma y_{6}^{2}\right)$ is independent of $y_{4} y_{6}$ and $y_{6}^{2}$, for all $k$. Then set $y_{8}^{\prime}$ $=y_{8}+\alpha y_{4}^{2}+\beta y_{2} y_{6}$ and define a new splitting $K$ such that $K\left(y_{8}^{\prime}\right)=J\left(y_{8}^{\prime}+\gamma y_{6}^{2}\right)$, $K\left(y_{i}\right)=J\left(y_{i}\right)$ otherwise. Then consider $M / M^{1}$, where $M^{1}$ is the ideal generated by $K\left(y_{8}^{\prime}\right)$, and in this manner the case $(2,3,4,6,8)$ is reduced to $(2,3,4,6)$. The verification that $\alpha, \beta$ and $\gamma$ exist in $Q_{2}$, provided $R^{4}(5) y_{2}=y_{6}+$ D.E. $\bmod 2$, is routine and is left to the reader. Therefore it remains to establish the above hypothesis.

We argue by contradiction. Suppose that $R^{4}(5) y_{2}=$ D.E. $\bmod 2$.

Considering $y_{6}$ in $F\left(2,5 ; y_{2}\right) \bmod 4$, deduce that $S^{2} y_{2}=$ D.E. $\bmod 4$ and so $S^{2} y_{2}=$ D.E.

Considering $y_{6}^{2}$ in $F\left(2,5 ; y_{6}\right) \bmod 16$, deduce that $S^{4} y_{6}=y_{4} y_{6}+$ I.E. $\bmod 2$.

Considering $y_{4} y_{8}$ in $F\left(2,5 ; y_{6}\right) \bmod 32$, deduce that $S^{6} y_{6}=2 y_{4} y_{8}+$ I.E. $\bmod 4$ if and only if $S^{2} y_{6}=2 y_{8}+$ D.E. $\bmod 4$.

Considering $y_{8}$ in $F\left(2,5 ; y_{4}\right) \bmod 32$, deduce that $S^{2} y_{6}=2 y_{8}+$ D.E. $\bmod 4$ if and only if $R^{4}(5) y_{4}=y_{8}+$ D.E. $\bmod 2$.

Considering $y_{8}^{2}$ in $F\left(2,5 ; y_{6}\right)$ mod 8 and using the last two necessary and sufficient conditions, obtain a contradiction.

$(2,4,6,7,8)$. The argument given for $(2,3,4,6,8)$, will imply that $R^{4}(5) y_{2}$ $=y_{6}+$ D.E. $\bmod 2$. Considering $y_{7}$ in $F\left(2,-1 ; y_{2}\right) \bmod 4$, obtain a contradiction.

This completes the proof of Theorem 5.15.

6. We now give the proofs of the results stated in $\S 1$.

Let $Y$ be a finite complex with $H_{*}(Y, Z)$ free of 2-torsion. Then $K\left(Y, Q_{2}\right)$ is a multiplicative $\psi^{k}$-module over $Q_{2}$ by Lemma 2.1 and Lemma 4.1. If $Y$ is not finite but has finite skeletons, we consider a skeleton of sufficiently high dimension, whose homology is free of 2-torsion, as all arguments are carried out at a finite stage in the cohomology. Then Theorem 1.4 follows from Theorem 3.3 and the remarks following Lemma 2.13 .

Theorem 1.3 is a consequence of Theorem 5.2, since if cohomology with $Z_{2}$ coefficients occurs in even dimensions only, the integral cohomology and homology are free of 2-torsion. The requirement in Theorem 5.2 that the height of the truncated polynomial algebra be exactly 3 is not significant, by the remarks before that theorem.

Theorem 1.2 likewise follows from Theorem 5.15.

In the proof of Theorem 1.1 , we use the $K^{*}$-theory of [11], complex $K$-theory with 
$Z_{2}$-grading. Let $Y$ be a finite complex with $H_{*}(Y, Z)$ free of 2-torsion; then the standard spectral sequence $H^{*}\left(Y, Q_{2}\right) \Rightarrow K^{*}\left(Y, Q_{2}\right)$ collapses, that is, if $G K^{*}\left(Y, Q_{2}\right)$ is the associated graded group of the filtered group $K^{*}\left(Y, Q_{2}\right)$, then $H^{*}\left(Y, Q_{2}\right) \cong G K^{*}\left(Y, Q_{2}\right)$. Further, this isomorphism preserves the ring structures. In particular

$$
\begin{gathered}
H^{2 n}\left(Y, Q_{2}\right) \cong \dot{K}_{2 n}^{0}\left(Y, Q_{2}\right) / K_{2 n+1}^{0}\left(Y, Q_{2}\right), \\
H^{2 n+1}\left(Y, Q_{2}\right) \cong K_{2 n+1}^{1}\left(Y, Q_{2}\right) / K_{2 n+2}^{1}\left(Y, Q_{2}\right),
\end{gathered}
$$

since

$$
K_{2 n}^{1}\left(Y, Q_{2}\right)=K_{2 n+1}^{1}\left(Y, Q_{2}\right) \quad \text { and } \quad K_{2 n+1}^{0}\left(Y, Q_{2}\right)=K_{2 n+2}^{0}\left(Y, Q_{2}\right) .
$$

Let $X$ be a connected finite complex and an $H$-space. Assume that $H_{*}(X, Z)$ is free of 2-torsion. As remarked earlier, the ring $H^{*}(X, Q)$ is an exterior algebra on odd-dimensional generators, and similarly so is $H^{*}\left(X, Q_{2}\right)$. It follows from the remarks above that $K^{*}\left(X, Q_{2}\right)$ is an exterior algebra on generators lying in $K^{1}\left(X, Q_{2}\right)$.

The existence of the $H$-map $m: X \times X \rightarrow X$ implies that $H^{*}\left(X, Q_{2}\right)$ and $K^{*}\left(X, Q_{2}\right)$ are graded Hopf algebras, $K^{*}\left(X, Q_{2}\right)$ having $Z_{2}$-grading. For the required Künneth formula in $K^{*}$-theory, we refer to [10]. If $H^{*}(X, Q)$ is primitive, then $H^{*}\left(X, Q_{2}\right)$ and $K^{*}\left(X, Q_{2}\right)$ are primitively generated Hopf algebras. One can see this by considering the maps induced by the coefficient inclusion $Q_{2} \rightarrow Q$,

$$
H^{*}\left(X, Q_{2}\right) \rightarrow H^{*}(X, Q) \text { and } K^{*}\left(X, Q_{2}\right) \rightarrow K^{*}(X, Q),
$$
where $K^{*}(X, Q)$ and $H^{*}(X, Q)$

are identified by the Chern character.

The Proof of Theorem 1.1. We consider $P_{2} X$, the projective plane of the $H$-space $X$. More precisely we consider the exact sequence given as (3.1) of [12]:

$$
\longrightarrow \tilde{h}^{q}(X) \stackrel{\phi}{\longrightarrow} \tilde{h}^{q}(X \times X) \stackrel{\lambda}{\longrightarrow} \tilde{h}^{q+2}\left(P_{2} X\right) \stackrel{i}{\longrightarrow} \tilde{h}^{q+1}(X),
$$

where $\phi=m^{*}-\pi_{1}^{*}-\pi_{2}^{*}, \pi_{i}: X \times X \rightarrow X, i=1$ or 2 , being the projections, and by $h^{a}()$ is meant either $H^{a}\left(, Q_{2}\right)$ or $K^{a}\left(, Q_{2}\right)$, the index in the second case being taken modulo 2 .

The proof given in [12] is for cohomology with coefficients in a field, but the proofs required above are very similar given that $H^{*}\left(X, Q_{2}\right)$ and $K^{*}\left(X, Q_{2}\right)$ are torsion free.

LEMMA 6.1. $H^{*}\left(P_{2} X, Q_{2}\right)$ is free.

Proof. This follows from the exact sequence (6.1). The proof is essentially that given as Lemma 3.3 in [14], using the additional information that $H^{*}\left(X, Q_{2}\right)$ is primitively generated as a Hopf algebra to deduce that $H^{\text {odd }}\left(P_{2} X, Q_{2}\right)$ is torsion free.

COROllaRY 6.2. $M=K\left(P_{2} X, Q_{2}\right)$ is a multiplicative $\psi^{k}$-module over $Q_{2}$. 
This follows from the lemma and Lemma 4.1.

Now let $P$ be the subspace spanned by the primitive elements in $h^{*}(X)$ and let $D$ be the subspace of decomposable elements. Then $h^{*}(X)=P \oplus D$, as all primitive elements are indecomposable.

Let $\hat{S}=D \otimes P \oplus P \otimes D \oplus D \otimes D$ in $h^{*}(X \otimes X)$ considered as a subset of $h^{*}(X \times X) \cong h^{*}(X) \otimes h^{*}(X)$. Set $S=\lambda(\hat{S})$.

Lemma 6.3. $h^{*}\left(P_{2} X\right)=A \oplus S$, where $A$ is a truncated polynomial algebra of height 3 on generators $u_{1}, u_{2}, \ldots, u_{n}$ such that $i\left(u_{1}\right), i\left(u_{2}\right), \ldots, i\left(u_{n}\right)$ form a multiplicative basis for $h^{*}(X)$ and $S$ is an ideal.

Proof. The proof of the corresponding result for $\mathrm{H}^{*}\left(P_{2} X, Q\right)$ and hence for $K^{*}\left(P_{2} X, Q\right)$ is given in [12]. This lemma can be deduced by embedding the sequence (6.1) with $h^{q}$ as $H^{q}\left(, Q_{2}\right)$ or $K^{q}\left(, Q_{2}\right)$ in the sequence (6.1) with $h^{q}$ as $H^{q}(, Q)$ or $K^{q}(, Q)$, as all groups are free.

The next two results apply only to $h^{q}(\quad)$ as $K^{q}\left(, Q_{2}\right)$.

LEMMA 6.4. $\psi^{k}(S) \subset S$, for each $k$.

Proof. $\psi^{k}(\hat{S}) \subset \hat{S}$ in $K^{*}\left(X * X, Q_{2}\right)$ as $\psi^{k}$ is a ring homomorphism. If $a \otimes b \in \hat{S}$, $\psi^{k}(\lambda(a \otimes b))=k \lambda\left(\psi^{k}(a \otimes b)\right)$, by the definition of $\lambda$ and Corollary 5.3 of [4]. The result follows.

COROLlaRY 6.5. $A$ is a multiplicative $\psi^{k}$-module over $Q_{2}$.

This now follows from Lemma 4.2.

Further it is clear that the graded ring corresponding to $A$ of Corollary 6.5 is just the $A$ of Lemma 6.3, when $h()=H\left(, Q_{2}\right)$. Now Theorem 1.1 follows from Theorem 5.15.

7. This paper has been concerned with the classification of truncated polynomial algebras of height 3 over $Q_{2}$, and the proofs have relied a great deal upon the "Integrality Condition" A3. However, it is not clear that this condition is at all essential for the results.

A second example of a multiplicative $\psi^{k}$-module over $Q_{2}$ closely connected with $K$-theory is that of a special $\lambda$-ring $R$ as defined by Grothendieck, whose associated graded ring with respect to the $\gamma$-filtration on $R, G_{y} R$ say, is torsion free. The verification of A3 can be carried out using a splitting theorem for special $\lambda$-rings proved in [17]. One therefore might consider the classification of such $\lambda$-rings where $R$ is a truncated polynomial algebra of height 3 over $Q_{2}$. However, the condition that $G_{y} R$ be torsion free is by itself very restrictive. Therefore we remove this condition and instead require that the indecomposable quotient of $G_{y} R$ modulo its torsion ideal, $G_{y} R /$ torsion, is torsion free. The dimensions of the generators of $R$ are defined to be the dimensions of the generators in this indecomposable quotient. If there are not more than two generators, it can be shown that their dimensions 
are the same as those given in Theorem 5.3 and Theorem 5.4. The proof however is much longer as there is no analogue of condition A3.

\section{REFERENCES}

1. J. F. Adams, On the non-existence of elements of Hopf invariant one, Ann. of Math. 72 (1960), 20-104.

2. - - H-spaces with few cells, Topology 1 (1962), 67-72.

3. - On Chern characters and the structure of the unitary group, Proc. Cambridge Philos. Soc. 57 (1961), 189-199.

4. — - Vector fields on spheres, Ann. of Math. 75 (1962), 603-632.

5. - On the groups $J(X)$. II, Topology 3 (1965), 137-172.

6. - On the groups $J(X)$. IV, Topology 5 (1966), 21-71.

7. J. F. Adams and M. F. Atiyah, $K$-theory and the Hopf invariant, Quart. J. Math. Oxford Ser. 17 (1966), 165-193.

8. M. F. Atiyah, Characters and cohomology of finite groups, Inst. Hautes Études Sci. Publ. Math. No. 9 (1961), 229-287.

9. - Power operations in K-theory, Quart. J. Math. Oxford Ser. 17 (1966), 165-193.

10. - Vector bundles and the Künneth formula, Topology 1 (1962), 245-248.

11. M. F. Atiyah and F. Hirzebruch, Vector bundles and homogeneous spaces, Proc. Sympos. Pure Math., Vol. 3, Amer. Math. Soc., Providence, R. I., 1961, pp. 7-38.

12. W. Browder and E. Thomas, On the projective plane of an $\mathrm{H}$-space, Illinois J. Math. 7 (1963), 492-502.

13. J. R. Hubbuck, Some results in the theory of H-spaces, Bull. Amer. Math. Soc. 74 (1968), 965-967.

14. - On homotopy commutative H-spaces, Topology (to appear).

15. I. M. James, On sphere bundles over spheres, Comment. Math. Helv. 35 (1961), 126-135.

16. N. E. Steenrod (edited by D. Epstein), Cohomology operations, Annals of Math. Studies, no. 50, Princeton Univ. Press, Princeton, N. J., 1962.

17. D. Tall, Thesis, Oxford University, 1967.

18. P. E. Thomas, Steenrod squares and H-spaces, Ann. of Math. 77 (1963), 306-317.

19. - Steenrod squares and H-spaces. II, Ann. of Math. 81 (1965), 473-495.

20. - On the mod 2 cohomology of certain H-spaces, Comment. Math. Helv. 37 (1962), 132-140.

21. C. T. C. Wall, Generators and relations for the Steenrod algebra, Ann. of Math. 72 (1960), 429-444.

Princeton University,

Princeton, New Jersey 